\title{
IMMERSIONS IN THE METASTABLE RANGE AND SPIN STRUCTURES ON SURFACES
}

\author{
TOBIAS EKHOLM
}

\section{Introduction.}

Recall that an immersion of a smooth manifold into another one is a map, which locally is a smooth embedding. Hence, smooth embeddings are immersions, but not vice versa. The basic classifications of immersions and embeddings are inspired by the spaces of immersions and embeddings: Two immersions (embeddings) are regularly homotopic (diffeotopic) if they can be connected by a path in the corresponding space. Smale and Hirsch [4] reduced the classification of immersions up to regular homotopy to homotopy theory. Haefliger [3] did the same for embeddings up to diffeotopy, in the metastable range.

One may consider embeddings up to regular homotopy, but the diffeotopy classification is more refined.

In this paper we consider immersions up to an equivalence, which is a generalization of diffeotopy. It is called diffeotopy equivalence. Two immersions are diffeotopy equivalent if they differ by diffeomorphisms, diffeotopic to identity, of the source and target.

We manage to classify generic immersions of a sufficiently highly connected manifold into Euclidean space, under the assumption that the dimensions are such that the dimension of the self intersection is 0,1 or 2 . The diffeotopy equivalence classes are described in terms of the topological type of the self intersection and numerical invariants of additional structures (e.g. spin structure), which appear on the self intersection. This description depends periodically on the dimension of the source manifold. When the dimension of the self intersection is 0 or 1 , the period is 2 . When it is 2 , the period is 4 .

The self intersection of a generic immersion in the metastable range is particularly simple. There, a generic immersion has no triple points and at a double point the intersection is transverse. This implies that the self inter- 
section is a submanifold of the target. When the target of a generic immersion in the metastable range is Euclidean space and the source is sufficiently connected, the diffeotopy equivalence class of this map is determined by the local (even infinitesimal) properties of it in a neighborhood of the preimage of the self intersection. This is the content of Theorem 1.2 below, which is the first step towards our classification.

As mentioned, there are structures induced on a self intersection. For example, if we let $F$ denote a component of a self intersection surface of a generic immersion then the preimage of $F$ is either disconnected or connected. In the former case the immersion induces a spin structure on $F$. There is a quadratic function on $H_{1}\left(F ; Z_{2}\right)$ associated to this spin structure. The Arf invariant of this function is preserved under diffeotopy equivalence. In the latter case the immersion induces a functional on immersed curves in $F$. The properties of it depend on the residue class of the dimension of the source manifold modulo 4 . We find invariants of this functional that are preserved under diffeotopy equivalence.

There is another application of the functional mentioned above: We use it to show that there are restrictions on the self intersection of a generic immersion, $f: V^{v} \rightarrow \mathrm{R}^{2 v-2}$, if $v \equiv 1 \bmod 4$. If $F$ is a nonorientable component of the self intersection, the genus of $F$ must be even (see Theorem 7.30). It is interesting that the same restriction holds for an immersion, without triple points and with transverse self intersection, of any orientable 3-manifold into the 4-sphere (see Theorem 7.31).

1.1. Isotopy equivalence. Let $f, h: X \rightarrow Y$ be maps of topological spaces. We say that $f$ is isotopy equivalent to $h$ if there exist isotopies $G_{t}: X \rightarrow X$ and $K_{t}: Y \rightarrow Y$ such that $G_{0}=1_{X}, K_{0}=1_{Y}$ and $K_{1} f G_{1}=h$. The analogous relations in the differential- and PL category will be called diffeotopy equivalence and PL isotopy equivalence respectively. Since our main concern will be the smooth case, all maps and manifolds are assumed to be smooth, unless something else is explicitly stated.

1.2. Immersions. A map $f: V^{v} \rightarrow W^{w}$ between two manifolds is called an immersion if $d f$, the differential of $f$, is injective at every point of $V$.

Definition 1.1. A generic immersion $f: V \rightarrow W$ is an immersion such that for any $z \in W$ the set $f^{-1}(z)$ contains at most two points and if $x, y \in V$, $x \neq y$ and $f(x)=f(y)=z$ then $d f T_{x} V+d f T_{y} V=T_{z} W$.

If $2 w>3 v$ (the metastable range) then any immersion $h: V^{v} \rightarrow W^{w}$ is homotopic, by an arbitrarily small homotopy, to a generic immersion.We write $\widetilde{M}_{f}=\left\{x \in V: f^{-1}(f(x)) \neq x\right\}$ and $M_{f}=f\left(\widetilde{M}_{f}\right)$. We call $M_{f}$ the self intersection and $\widetilde{M}_{f}$ the double locus. 
1.3. Statements of the main results. Let $f, h: V^{v} \rightarrow W^{w}$ be generic immersions. As already mentioned, if $f$ and $h$ are diffeotopy equivalent then $M_{f} \approx M_{h}$ and $\widetilde{M}_{f} \approx \widetilde{M}_{h}$ (where " $\approx$ " denotes "is diffeomorphic to").

THEOREM 1.2. Let $f, h: V^{v} \rightarrow \mathbf{R}^{w}$ be generic immersions. Let $w=v+k$, where $k \geq 3$ and $v+3<2 k \leq 2 v$. Assume that $V$ is closed and $(v-k+1)$ connected and that there are diffeotopies $K_{t}: \mathrm{R}^{w} \rightarrow \mathrm{R}^{w}$ and $G_{t}: V \rightarrow V$ such that $K_{1} f G_{1}|T=h| T$, where $T$ is a tubular neighborhood of $\widetilde{M}_{h}$. Then $f$ is diffeotopy equivalent to $h$.

A proof is presented in Section 3.

If $f: V^{v} \rightarrow \mathrm{R}^{2 v}$ is a generic immersion then $M_{f}$ is a collection of points. If $V$ and $\mathrm{R}^{2 v}$ are oriented and $v$ is even then there is an induced orientation of $M_{f}$. Let $c \subset M_{f}$ be a component (i.e. $c$ is a point in $M_{f}$ ). When $v$ is even, we let $s(c) \in Z_{2}$ be the signature of $c$. When $v$ is odd, there are no local obstructions to diffeotopy equivalence and we let $s(c)=0$.

TheOREM 1.3. Let $f, h: V^{v} \rightarrow \mathrm{R}^{2 v}, v \geq 4$, be generic immersions. Assume that $V$ is closed and simply connected. Then $f$ is diffeotopy equivalent to $h$ if and only if there is a 1-1 correspondence between the components $C_{f}$ of $M_{f}$ and $C_{h}$ of $M_{h}$ such that if $C_{f}$ corresponds to $C_{h}$ then $s\left(C_{f}\right)=s\left(C_{h}\right)$, where $s$ is as described above.

Theorem 1.3 is proved in Section 4.2. There is an analogue of Theorem 1.3 in the PL category that actually is somewhat stronger. We can replace $v \geq 4$ above by $v \geq 3$. For the necessary definitions in the PL case, see Section 4.3.

If $f: V^{v} \rightarrow \mathrm{R}^{2 v-1}$ is a generic immersion then $M_{f}$ is a collection of circles. Assume that $V$ is a spin manifold and let $c$ be a component of $M_{f}$. We shall define an invariant $s(c) \in \mathbf{Z}_{2}$, which describes the local obstructions to diffeotopy equivalence. The preimage $\widetilde{c}=f^{-1}(c)$ has either one or two components. If $\widetilde{c}$ is disconnected then there is an induced spin structure on $c$ (cf. Proposition 2.10) and, in this case, we let $s(c)=1$ if the induced spin structure is trivial (spin cobordant to zero) and $s(c)=0$ otherwise. If $\widetilde{c}$ is connected then, given a framing of the normal bundle of $\widetilde{c}$, the map $f$ induces a structure similar to a framing of the normal bundle of $c$. Such a structure will be called a twist framing (see Definition 5.3). There are two twist framings up to isotopy. Taking into account also the framing from which the twist framing is induced, it is possible to define $s(c)$ (see Definition 6.7) also in this case. The latter case, $\widetilde{c}$ is connected for some component $c$ of $M_{f}$, appears only if $v=\operatorname{dim} V$ is odd.

TheOrem 1.4. Let $f, h: V^{v} \rightarrow \mathrm{R}^{2 v-1}, v \geq 6$, be generic immersions. Assume that $V$ is closed and 2-connected. Then $f$ is diffeotopy equivalent to $h$ if and 
only if there is a 1-1 correspondence between the components $C_{f}$ of $M_{f}$ and $C_{h}$ of $M_{h}$ such that if $C_{f}$ corresponds to $C_{h}$ then $f^{-1}\left(C_{f}\right)$ and $h^{-1}\left(C_{h}\right)$ have the same number of components and $s\left(C_{f}\right)=s\left(C_{h}\right)$, where $s$ is as described above.

Theorem 1.4 is proved in Section 6.2.

If $f: V^{v} \rightarrow \mathrm{R}^{2 v-2}$ is a generic immersion then $M_{f}$ is a collection of closed surfaces. Assume that $V$ is a spin manifold and let $F$ be a component of $M_{f}$. Let $g(F)=\operatorname{genus}(F)$. We shall define an invariant $s(F) \in G(n,[v])$, where the group $G(n,[v])$ depends on the number $n$ of components of $\widetilde{F}=f^{-1}(F)$ and the residue class $[v]$ of $v$ modulo 4 in the following way, $G(2,[v])=\mathrm{Z}_{2}$ for all $[v]$ and $G(1,[v])=0,0, \mathbf{Z}_{2} \oplus \mathbf{Z}_{2}, \mathbf{Z}_{8}$ for $[v]=0,1,2,3$ respectively. The invariant $s(F)$ describes the local obstructions to diffeotopy equivalence and is defined in Table 1 below. The first row in this table is straightforward to explain: If $\widetilde{F}$ has two components then $f$ induces a spin structure on $F$ and hence a $Z_{2}$-quadratic form $q_{f, F}$ on $H_{1}\left(F ; Z_{2}\right)$. Let $\operatorname{Arf}\left(q_{f, F}\right)$ denote the Arf invariant of the quadratic function $q_{f, F}$ and define $s(F)=\operatorname{Arf}\left(q_{f, F}\right) \in \mathbf{Z}_{2}$, in this case. The other rows should be read in a similar way. The second and third row are explained in Section 7.6, the fourth row in Section 7.5 and the fifth row in Section 7.4.

\begin{tabular}{|c|c|c|c|c|c|}
\hline$n$ & {$[v]$} & $F$ & Local obstruction & $G(n,[v])$ & $s(F)$ \\
\hline 2 & $\begin{array}{c}0 \\
1,2 \\
3\end{array}$ & Orientable & $\begin{array}{c}\text { A spin structure on } F \\
\text { and hence a } \mathrm{Z}_{2} \text {-quadratic } \\
\text { function } q_{f, F} \text { on } H_{1}\left(F ; Z_{2}\right)\end{array}$ & $\mathrm{Z}_{2}$ & $\operatorname{Arf}\left(q_{f, F}\right)$ \\
\hline 1 & 0 & Orientable & None & 0 & 0 \\
\hline 1 & 1 & $\begin{array}{c}\text { Non- } \\
\text { orientable, } \\
g(f) \\
\text { even }\end{array}$ & $\begin{array}{c}\text { The quadratic function } \\
\text { of a spin structure } \\
\text { evaluated at } b \in H_{1}\left(\widetilde{F} ; \mathbf{Z}_{2}\right) \text {, } \\
\text { with } f_{*}(b) \text { dual } \\
\text { to } w_{1} \in H^{1}\left(F ; Z_{2}\right)\end{array}$ & $\mathrm{Z}_{2}$ & $q_{f, \widetilde{F}}(b)$ \\
\hline 1 & 2 & $\begin{array}{l}\text { Orien- } \\
\text { table }\end{array}$ & $\begin{array}{c}\mathrm{A} \mathrm{Z}_{2} \oplus \mathrm{Z}_{2}-\text { quadratic } \\
\text { function } q_{f, F} \text { on } H_{1}\left(F ; \mathrm{Z}_{2}\right)\end{array}$ & $\mathbf{Z}_{2} \oplus \mathbf{Z}_{2}$ & $\operatorname{Arf}\left(q_{f, F}\right)$ \\
\hline 1 & 3 & $\begin{array}{l}\text { Non- } \\
\text { orien- } \\
\text { table }\end{array}$ & $\begin{array}{c}\text { A pin structure on } F \\
\text { and hence a } \mathrm{Z}_{4} \text {-quadratic } \\
\text { function } q_{f, F} \text { on } H_{1}\left(F ; Z_{2}\right)\end{array}$ & $\mathrm{Z}_{8}$ & $\begin{array}{l}\text { Brown's } \\
\text { invariant } \\
\text { of } q_{f, F}\end{array}$ \\
\hline
\end{tabular}

TABLE 1. Local obstructions to diffeotopy equivalence associated to a component $F$ of a self intersection surface.

TheORem 1.5. Let $f, h: V^{v} \rightarrow \mathrm{R}^{2 v-2}, v \geq 8$, be generic immersions. Assume that $V$ is closed and 3-connected. Then $f$ and h are diffeotopy equivalent if and only if there is a 1-1 correspondence between the components $C_{f}$ of $M_{f}$ and $C_{h}$ 
of $M_{h}$ such that if $C_{f}$ corresponds to $C_{h}$ then $f^{-1}\left(C_{f}\right)$ and $h^{-1}\left(C_{h}\right)$ have the same number of components, $g\left(C_{f}\right)=g\left(C_{h}\right)$ and $s\left(C_{f}\right)=s\left(C_{h}\right)$, where $s$ is as described in Table 1.

Theorem 1.5 is proved in Section 7.7.

\section{Self intersection and double locus of a generic immersion.}

Recall our definition of generic immersion (Definition 1.1). If we are not in the metastable range then the term generic is somewhat misleading. For simplicity, we keep this notation also outside the metastable range.

Proposition 2.1. Let $f: V^{v} \rightarrow W^{w}$ be a generic immersion of a closed manifold into a manifold. Then $M_{f}$ is a closed submanifold of $W$ and $\widetilde{M}_{f}$ is a closed submanifold of $V$. Moreover, $f \mid \widetilde{M}_{f}: \widetilde{M}_{f} \rightarrow M_{f}$ is a (possibly disconnected) double cover of $M_{f}$.

Definition 2.2. Let $f: V^{v} \rightarrow W^{w}$ be a generic immersion. We denote by $M_{f}^{\prime}$ the union of those components $C_{f}$ of $M_{f}$ such that $f^{-1}\left(C_{f}\right)$ is connected. Let $M_{f}^{\prime \prime}=M_{f}-M_{f}^{\prime}$.

\subsection{Orientation.}

Proposition 2.3. Let $f: V^{v} \rightarrow W^{w}$ be a generic immersion of an orientable closed manifold into an orientable manifold. If $w-v$ is even then $M_{f}$ and $\widetilde{M}_{f}$ are orientable.

Proof. Since $\widetilde{M}_{f}$ is a double cover of $M_{f}$ it suffices to prove that $M_{f}$ is orientable. Let $x \in M_{f}$. Near $x$, the submanifold $M_{f}$ is the intersection of two oriented submanifolds $U_{1}$ and $U_{2}$ of dimension $v$. If we order these, there is a standard way to assign an orientation to a neighborhood of $x$ in $M_{f}$. Since $w-v$ is even, this orientation does not depend on the ordering.

REMARK 2.4. If $V$ and $W$ in Proposition 2.3 are oriented then there is an induced orientation of $M_{f}$.

Proposition 2.5. Let $f: V^{v} \rightarrow W^{w}$ be a generic immersion of an orientable closed manifold into an orientable manifold. If $w-v$ is odd then the covering $\widetilde{M}_{f} \rightarrow M_{f}$ is the orientation double covering.

Proof. Let $p \in M_{f}$ and $f^{-1}(p)=\left\{p_{1}, p_{2}\right\}$. There are neighborhoods $N \subset M_{f}, N_{1} \subset V$ and $N_{2} \subset V$ of $p, p_{1}$ and $p_{2}$, respectively such that $N=f\left(N_{1}\right) \cap f\left(N_{2}\right)$. Ordering $N_{1}$ and $N_{2}$ we get an induced orientation of $N$. We can pull back this orientation to $f^{-1}(N) \cap N_{1}=\widetilde{N}_{1}$. Changing the ordering we pull back the opposite orientation to $\widetilde{N}_{2}$. It follows that $\widetilde{M}_{f}$ is the orientation double cover. 
REMARK 2.6. If $f: V^{v} \rightarrow W^{w}$ is a generic immersion of orientable manifolds then $\widetilde{M}_{f}$ is orientable.

2.2. Spin. Let $\eta$ be an orientable vector bundle over a base space $B$. Let $E(\mathrm{SO}(\eta))$ denote the total space of its principal SO-bundle. Aspin structure on $\eta$ can be viewed as an element in the cohomology group $H^{1}\left(E(\mathrm{SO}(\eta)) ; \mathrm{Z}_{2}\right)$, which takes the value 1 on the fiber class, i. e. the class represented by inclusion of the nontrivial loop in a fiber. In the special case when the vector bundle is 1-dimensional we consider the spin structure as an element in $H^{1}\left(B ; Z_{2}\right)$. A spin structure on an orientable manifold is a spin structure on its tangent bundle. The following lemma is proved in [11].

Lemma 2.7. Let $\eta^{n}, \eta^{m}, \eta^{k}$ be orientable vector bundles over B. Let $n=m+k$ and $\eta^{n}=\eta^{m} \oplus \eta^{k}$. Spin structures on any two of these bundles determine a spin structure on the third.

As a consequence of Lemma 2.7 we get the spin analogue of Remark 2.6:

Proposition 2.8. Let $f: V^{v} \rightarrow W^{w}$ be a generic immersion of spin manifolds. Then $\widetilde{M}_{f}$ is spin.

Proof. Let $i: \widetilde{M}_{f} \rightarrow \widetilde{M}_{f}$ denote the involution (i.e. $f(i(x))=f(x)$, $x \in \widetilde{M}_{f}$ ). Since $f$ is a generic immersion, we have the following splitting

$$
\left(f^{*} T W\right)\left|\tilde{M}_{f}=T V\right| \widetilde{M}_{f} \oplus i^{*} N\left(\widetilde{M}_{f} \subset V\right),
$$

where $N\left(\widetilde{M}_{f} \subset V\right)$ denotes the normal bundle of $\widetilde{M}_{f}$ in $V$.An application of Lemma 2.7 shows that $i^{*} N\left(\widetilde{M}_{f} \subset V\right)$ is a spin bundle. The decomposition

$$
T V \mid \widetilde{M}_{f}=N\left(\widetilde{M}_{f} \subset V\right) \oplus T \widetilde{M}_{f},
$$

then shows that $\widetilde{M}_{f}$ is spin.

Proposition 2.9. Let $X$ and $Y$ be transversely intersecting submanifolds of a manifold $W$. Let $Z=X \cap Y$. If there are spin structures on $X, Y$ and $W$ then there is an induced spin structure on $Z$.

Proof. The restriction of the tangent bundle of $W$ to $Z$ decomposes as follows:

$$
\begin{aligned}
& T W|Z=T X| Z \oplus N(Z \subset Y) \\
& T W|Z=T Y| Z \oplus N(Z \subset X) \\
& T W \mid Z=N(Z \subset X) \oplus N(Z \subset Y) \oplus T Z .
\end{aligned}
$$

Apply Lemma 2.7.

Proposition 2.10. Let $f: V^{v} \rightarrow W^{w}$ be a generic immersion. Let $C_{f}$ be a 
component of $M_{f}$ such that $f^{-1}\left(C_{f}\right)=\widetilde{C}_{f}$ is disconnected. If there are spin structures on $V$ and $W$ then there is an induced spin structure on $C_{f}$.

Proof. There are disjoint tubular neighborhoods $N_{1}$ and $N_{2}$ of the components of $\widetilde{C}_{f}$ such that $f \mid N_{i}$ is an embedding $i=1,2$. Now, $C_{f}$ is the transverse intersection of the two embedded spin submanifolds $f\left(N_{1}\right)$ and $f\left(N_{2}\right)$ in the spin manifold $W$.

Spin structures are defined on oriented manifolds. The self intersection in Proposition 2.10 receives an orientation if we order $N_{1}$ and $N_{2}$ in the proof. If $w-v$ is even the induced orientation is independent of the ordering. If $w-v$ is odd and this ordering is changed then the orientation changes and the spin structure is reversed.

If $M$ is a spin manifold and $\vec{c}$ is a framed loop in $M$, we can orthonormalize the framing and regard $\vec{c}$ as a loop in the principal bundle. Then $\vec{c}$ represents a homology class $[\vec{c}] \in H_{1}\left(E(\mathrm{SO}(T M)) ; \mathrm{Z}_{2}\right)$. Let $X, Y, Z$ and $W$ be as in Proposition 2.9. Let $\vec{c}_{Z}$ be a framed loop in $Z$. If we extend this framing of $T Z$ along $c$ to framings of $T X$ and $T Y$ then we obtain two framed curves $\vec{c}_{X}$ and $\vec{c}_{Y}$ in $X$ and $Y$ respectively. Taking the sum of the extensions and the framing of $T Z$ we get a framing of $T W$ along $c$. The framed curve in $W$, obtained in this way, will be denoted $\vec{c}_{W}$.

Proposition 2.11. Let $\xi_{X}, \xi_{Y}$ and $\xi_{W}$ denote the spin structures on $X, Y$ and $W$ respectively. Let $\xi_{Z}$ denote the spin structure induced on $Z$. Then

$$
\left\langle\xi_{Z},\left[\vec{c}_{Z}\right]\right\rangle=\left\langle\xi_{X},\left[\vec{c}_{X}\right]\right\rangle+\left\langle\xi_{Y},\left[\vec{c}_{Y}\right]\right\rangle+\left\langle\xi_{W},\left[\vec{c}_{W}\right]\right\rangle .
$$

Proof. This follows from the proof of Lemma 2.7, given in [11].

REMARK 2.12. Assume that the extension to $T X$ is changed in such a way that the fiber class of $\mathrm{SO}(T X)$ is added to $\left[\vec{c}_{X}\right]$, and that $\left[\vec{c}_{Y}\right]$ is fixed. Then $\left[\vec{c}_{W}\right]$ changes by the fiber class in $\operatorname{SO}(T W)$. Hence, the formula in Proposition 2.11 is independent of the extensions of the framing of $T Z$.

Remark 2.13. If the manifold $Z$ in Proposition 2.11 happens to be $S^{1}$ we see that $\xi_{Z}$ is an element in $H^{1}\left(S^{1} ; Z_{2}\right)$. For future reference we note that there are two spin structures on the circle. The trivial spin structure, which is induced from the unique spin structure on the disk and corresponds to the cohomology class $\xi$ which takes the value 1 on the generator of $H_{1}\left(S^{1} ; Z_{2}\right)$. The Lie group one, which corresponds to the 0 cohomology class and with which $S^{1}$ is not a spin boundary.

2.3. Straightening. We can straighten a generic immersion close to its self intersection, using a diffeotopy of the target space. More precisely, consider a generic immersion $f: V^{v} \rightarrow W^{w}$. Let $\xi^{w-v}$ and $\eta^{2 v-w}$ be vector bundles over 
$\widetilde{M}_{f}$ and $M_{f}$, respectively. Denote their total spaces $E(\xi)$ and $E(\eta)$. Let $\phi: E(\xi) \rightarrow U \subset V$ and $\psi: E(\eta) \rightarrow N \subset W$ be embeddings. That is, $U$ and $N$ are tubular neighborhoods of $\widetilde{M}_{f}$ and $M_{f}$, respectively. Assume that $f(U) \subset N$.

LEMma 2.14. Under the conditions above there are open disk subbundles $\xi^{\prime} \subset \xi, \eta^{\prime} \subset \eta$ and a diffeotopy $K_{t}: W \rightarrow W$ such that $\psi^{-1} K_{1} f \phi: E\left(\xi^{\prime}\right) \rightarrow E\left(\eta^{\prime}\right)$ is a vector bundle map covering $f: \widetilde{M}_{f} \rightarrow M_{f}$.

The proof of Lemma 2.14 is a standard application of the techniques used to prove e.g. the Tubular Neighborhood Theorem. Note that if $\xi^{w-v}=$ $N\left(\widetilde{M}_{f} \subset V\right)$ and $\eta^{2 v-w}=N\left(M_{f} \subset W\right)$, Lemma 2.14 tells us that any generic immersion $f: V \rightarrow W$ is diffeotopy equivalent to a map that equals the normal derivative of $f$ in a neighborhood of the self intersection.

\section{Proof of Theorem 1.2.}

Using Lemma 2.14 we can find a diffeotopy $R_{t}: \mathrm{R}^{w} \rightarrow \mathrm{R}^{w}$ that is fixed outside a neighborhood of $h(T)$ and tubular neighborhoods $N \subset T$ of $\widetilde{M}_{h}$ and $U$ of $M_{h}$ such that $R_{1} h: N \rightarrow U$ and $R_{1} h: \partial N \rightarrow \partial U$. Let $h^{\prime}=R_{1} h$ and $f^{\prime}=R_{1} K_{1} f G_{1}$. Then $f^{\prime}\left|N=h^{\prime}\right| N$. Assume that there is a diffeotopy $L_{t}^{\prime}: \mathrm{R}^{w}-U \rightarrow \mathrm{R}^{w}-U$, keeping $\partial U$ fixed and such that $L_{1}^{\prime} f^{\prime} \mid(V-T)=$ $h^{\prime} \mid(V-T)$. Then, clearly, $f$ is diffeotopy equivalent to $h$. Thus, to complete the proof it is enough to prove that $f^{\prime}, h^{\prime}: V-T \rightarrow \mathrm{R}^{w}-U$ are ambient diffeotopic keeping $\partial U$ fixed. First of all it is necessary that $f^{\prime}$ and $h^{\prime}$ are homotopic relative $\partial T$. They are both solutions to the lifting problem

$$
\begin{array}{ccc}
\partial T & \rightarrow & \mathrm{R}^{w}-U \\
\downarrow & \nearrow & \\
V-T & &
\end{array}
$$

The obstructions to a homotopy are found in

$$
H^{r}\left(V-T, \partial T ; \pi_{r}\left(\mathrm{R}^{w}-U\right)\right) \cong H_{v-r}\left(V-T ; \pi_{r}\left(\mathrm{R}^{w}-U\right)\right) .
$$

Now, $\mathrm{R}^{w}-U$ is a deformation retract of $\mathrm{R}^{w}-M_{h}$. Since $M_{h}$ is a $(v-k)$-dimensional submanifold, we see by transversality that $\mathrm{R}^{w}-U$ is at least $(v+k-1)-(v-k)-1=(2 k-2)$ connected. By assumption $2 k-2>v$ and thus all obstructions vanish. Let $F^{\prime}:(V-T) \times I \rightarrow \mathrm{R}^{w}-U$ denote the homotopy between $f^{\prime}$ and $h^{\prime}$, fixing $\partial T$. Define $F^{\prime \prime}:(V-T) \times$ $I \rightarrow\left(\mathrm{R}^{w}-U\right) \times I$ by

$$
F^{\prime \prime}(v, t)=\left(F^{\prime}(v, t), t\right) .
$$

Using smooth approximation we can assume that $F^{\prime \prime}$ is smooth and equals $f^{\prime}, h^{\prime}$ at the top and bottom. Choose a smooth triangulation of $\mathrm{R}^{w}-U$. We 
may assume that $f^{\prime}$ and $h^{\prime}$ are PL embeddings relative this triangulation (at least they are isotopic to such maps and this is what is really needed). By Theorem 3.1 and Remark 3.2 below we can conclude that $F^{\prime \prime}$ is homotopic, keeping the boundary fixed, to a PL embedding, provided some connectedness conditions are fulfilled. More precisely, we shall verify that

(a) $V-T$ is $2(v+1)-(v+k+1)=v-k+1$ connected,

(b) $\mathrm{R}^{w}-U$ is $v-k+2$ connected,

(c) $v+k-v=k \geq 3$.

Condition (c) holds by assumption. Condition (b) holds by the above since $v-k+2<2 k-2$. To see that condition (a) follows from our assumptions, note that $V-T$ is a deformation retract of $V-\widetilde{M}_{h}$. Hence, $V-T$ is $(v-k+1)$ connected by general position and the fact that $V$ is $(v-k+1)$ connected. Thus, there is a PL embedding

$$
F:(V-T) \times I \rightarrow\left(\mathrm{R}^{w}-U\right) \times I,
$$

such that $F_{0}=f^{\prime}$ and $F_{1}=h^{\prime}$. Adjoining copies of $(V-T) \times[0, \epsilon]$ at the ends of $(V-T) \times I$ we can make $F_{s}=f^{\prime}$ for $s$ near 0 and similarly at the other end. Hence $F:(V-T) \times I \rightarrow\left(\mathrm{R}^{w}-U\right) \times I$ is an allowable concordance connecting $f^{\prime}$ and $h^{\prime}$. Proposition 3.3 then implies that $f^{\prime}$ and $h^{\prime}$ are PL isotopic (even ambient isotopic). We conclude from Theorem 3.5 that $f^{\prime}$ is smoothly isotopic to $h^{\prime}$, keeping $\partial T$ fixed, since $2(v+k)>3(v+1)$. Proposition 3.4 shows that this differentiable isotopy extends to an ambient diffeotopy that keeps $\partial U$ fixed. Thus we have the required diffeotopy $L_{t}$ discussed in the beginning of the proof.

We state below the theorems used in the above proof.

THeOREM 3.1. (Hudson) Let $M$ and $Q$ be connected PL manifolds of dimensions $m$ and $q$ respectively, $M$ being compact. Let $f: M \rightarrow Q$ be a map such that $f^{-1}(\partial Q)=\partial M$, and the restriction $\left.f\right|_{\partial M}$ is a PL embedding. If $q-m \geq 3$,

$$
\pi_{r}(f)=0 \quad \text { for } r \leq 2 m-q+1,
$$

and

$$
\pi_{r}(M)=0 \quad \text { for } r \leq 3 m-2 q+2,
$$

then $f$ is homotopic, keeping $\partial M$ fixed, to a PL embedding. Here, $\pi_{r}(f)$ denotes the $r^{\text {th }}$ relative homotopy group of $\left(I_{f}, M\right)$ where $I_{f}$ is the mapping cylinder of $f$.

Theorem 3.1 is proved in [6].

Remark 3.2. The exact homotopy sequence of the pair $\left(I_{f}, M\right)$ shows that $\pi_{r}(f)=0$ if $\pi_{r-1}(M)=0$ and $\pi_{r}(Q)=0$. 
Proposition 3.3. (Hudson) Let $f, h: M \rightarrow Q$ be allowably concordant PL embeddings keeping $Y \subset M$ fixed. Suppose that $M$ is compact, $f^{-1}(\partial Q) \subset$ $N \subset Y$ and $\operatorname{dim}(Q)-\operatorname{dim}(M) \geq 3$. Then $f$ and $h$ are ambient isotopic keeping $f Y \cup \partial Q$ fixed.

Proposition 3.3 is proved in [5].

Proposition 3.4. Let $f, h: M \times I \rightarrow Q \times I$ be allowably concordant smooth embeddings keeping $N$ fixed. Suppose that $M$ is compact, $f^{-1} \partial Q=N$ and $\operatorname{dim}(Q)-\operatorname{dim}(M) \geq 3$. Then $f$ is ambient diffeotopic to $h$ keeping $\partial Q$ fixed.

Remark. Proposition 3.4 follows from Theorem 2.1 in [5] in the same way as Proposition (named Corollary 1.3 in [5]) follows from Theorem 1.1 in this paper.

THeOREm 3.5. (Haefliger) Let $f_{0}, f_{1}: M^{m} \rightarrow Q^{q}$ be two differentiable embeddings. Any isotopy connecting $f_{0}$ and $f_{1}$ can be approximated by a differentiable isotopy connecting $f_{0}$ and $f_{1}$ if $2 q>3(m+1)$.

See [1] for reference.

\section{0-dimensional self intersection.}

A generic immersion $f: V^{v} \rightarrow W^{2 v}$ has 0 -dimensional self intersection. If $V$ is closed, Proposition 2.1 shows that $M_{f}$ is a finite collection of points. If $v$ is even and $V$ and $W$ are oriented, Proposition 2.3 shows that there is an induced orientation of $M_{f}$.

\subsection{The invariants.}

Definition 4.1. Let $f: V^{v} \rightarrow \mathrm{R}^{2 v}$ be a generic immersion. Let $c$ be a point in $M_{f}$. Assume that $V$ and $\mathrm{R}^{2 v}$ are oriented.

(a) If $v$ is even, define $s(c)=0$ if the induced local orientation of $\mathrm{R}^{2 v}$ at $c$ agrees with that of $\mathrm{R}^{2 v}$ and $s(c)=1$ if it does not.

(b) If $v$ is odd, define $s(c)=0$.

Lemma 4.2. Let $f: V^{v} \rightarrow W^{2 v}$ be a generic immersion. Assume that $V$ and $W$ are oriented. Let $K_{t}: W^{2 v} \rightarrow W^{2 v}$ and $G_{t}: V^{v} \rightarrow V^{v}$ be diffeotopies. If $c \in M_{f}$ then $K_{1} c \in M_{K_{1} f G_{1}}$ and $s(c)=s\left(K_{1} c\right)$.

Proof. Diffeotopies preserve local orientation.

4.2. Proof of Theorem 1.3. The only if part follows from Lemma 4.2 and the fact that the diffeomorphism type of the self intersection is preserved under diffeotopy equivalence. Orient $V$ and $\mathrm{R}^{2 v}$. Let $z \in M_{f}$ and $\zeta \in M_{h}$. Assume that $s(z)=s(\zeta)$. Suppose that $f^{-1}(z)=\{x, y\}$ and $h^{-1}(\zeta)=\{\xi, \eta\}$. 
When $v$ is odd we get an induced orientation of $z$ by ordering $\{x, y\}$. Changing the ordering changes the induced orientation. Assume that the notation is chosen so that $(x, y)$ induces the same orientation on $z$ as does $(\xi, \eta)$ on $\zeta$. Choose diffeotopies $G_{t}: V \rightarrow V$ and $K_{t}^{\prime}: \mathrm{R}^{2 v} \rightarrow \mathrm{R}^{2 v}$ such that $G_{1}(\xi)=x$, $G_{1}(\eta)=y$ and $K_{1}^{\prime}(z)=\zeta$. Then $K_{1}^{\prime} f G_{1}(\xi)=K_{1}^{\prime} f G_{1}(\eta)=\zeta$. By Lemma 2.14 we can, locally, replace the maps $h$ and $K_{1}^{\prime} f G_{1}$ by their normal derivatives at $\{\xi, \eta\}$. Pick framings of $N(\xi \subset V)$ and $N(\eta \subset V)$. Then $h$ and $K_{1}^{\prime} f G_{1}$ induce two framings of $N\left(\zeta \subset \mathrm{R}^{2 v}\right)$. By our assumptions the orientations of these framings agree. It is then easy to find adiffeotopy $K_{t}^{\prime \prime}: \mathrm{R}^{2 v} \rightarrow \mathrm{R}^{2 v}$, supported in a neighborhood $N$ of $\zeta$, such that $K_{1}^{\prime \prime} K_{1}^{\prime} f G_{1}|U=h| U$ where $U$ is some neighborhood of $\{\xi, \eta\}$. Repeating this procedure for all points in $M_{h}$ we find diffeotopies $L_{t}: V \rightarrow V$ and $K_{t}: \mathrm{R}^{2 v} \rightarrow \mathrm{R}^{2 v}$ such that $K_{1} f L_{1}|W=h| W$, where $W$ is a neighborhood of $\widetilde{M}_{h}$. An application of Theorem 1.2 finishes the proof.

4.3. PL results. A PL immersion is a PL $\operatorname{map} f: V^{v} \rightarrow W^{w}$ that locally, is a locally flat PL embedding. (If $w-v \geq 3$, local flatness is automatic.) If $w=2 v$ then we say that the PL immersion $f$ is generic if any self intersection point is an intersection of two $v$-dimensional faces, of $f(V)$, in general position. Theorem 1.2 can be proved in the PL case. The proof is exactly the same as that given for the smooth case. However, we need not apply Theorem 3.5 and therefore we can weaken the assumption $v+3<2 k$ to $v+3 \leq 2 k$. (Haefliger's construction of knotted differential 3-spheres in 6space (cf. [2]) shows that the condition $v+3 \leq 2 k$ is not sufficient in the smooth category.) We can then prove Theorem 1.3 in the PL category with the assumption $v \leq 4$ replaced by $v \leq 3$. It is only a matter of finding the PL counterparts of the smooth concepts used in the above proof. We give a brief description: Let $h: V^{v} \rightarrow \mathrm{R}^{2 v}$ be a generic PL immersion. If $z \in M_{h}$ then $z$ is the intersection of two $v$-disks $D_{1}$ and $D_{2}$ in general position. We can find a $2 v$-disk $D$ such that $D_{1} \cup D_{2} \subset D$ and $\partial D_{1} \cup \partial D_{2} \subset \partial D$. Now, $s(z)=l k\left(\partial D_{1}, \partial D_{2}\right)$, if $v$ is even, where the linking number is computed in $\partial D$. Since $v \geq 3$ this linking number determines the isotopy class of the link.

Probably there are PL versions of Theorems $1.4,1.5$ as well. To translate the proofs to the PL case one must study the PL analogue of the smooth normal bundle of the self intersection.

\section{Framed and twist framed circles in $R^{n}$.}

If $c$ is a circle in the self intersection of a generic immersion there is some induced structure in the normal bundle of $c$. We study the structures that arise in this way when the dimension of the self intersection is 1 or 2 . 
5.1. Framed circles. Let $c: S^{1} \rightarrow \mathrm{R}^{n}, n \geq 4$. Let $x_{i}: S^{1} \rightarrow N\left(c \subset \mathrm{R}^{n}\right)$, $i=1, n-1$ be such that $X=\left(x_{1}, x_{n-1}\right)$ is a framing of the normal bundle of $c$. Adding the tangent vector $\dot{c}$ to this framing we obtain a framing $(\dot{c}, X)$ of $T \mathrm{R}^{n} \mid c$, if the orientation of $(\dot{c}, X)$ does not agree with that of $\mathrm{R}^{n}$ we consider instead $(-\dot{c}, X)$. We denote this framed curve $\vec{c}$. As in Section 2.2, $\vec{c}$ defines an element (after orthonormalization) $[\vec{c}] \in H_{1}\left(\mathrm{SO}\left(T \mathrm{R}^{n}\right) ; \mathrm{Z}_{2}\right)$. Since $H^{1}\left(\mathrm{R}^{n}, \mathrm{Z}_{2}\right)=0$, there is a unique spin structure $\xi$ on $\mathrm{R}^{n}$. Two framed framed circles $\vec{b}$ and $\vec{c}$ are framed isotopic if and only if $\langle\xi,[\vec{b}]\rangle=\langle\xi,[\vec{c}]\rangle$. We may also view the vectors in the frame $(\dot{c}, X)$ as column vectors of a matrix. In this way, we get a loop $\sigma(\vec{c})$ in $\mathrm{SO}(n)$. Two framed circles $\vec{b}$ and $\vec{c}$ are framed isotopic if and only if $\sigma(\vec{b}) \simeq \sigma(\vec{c})$.

5.2. Paths and loops in $\mathrm{SO}(n)$ Let $\mathbf{I}$ denote the identity matrix.

Definition 5.1. If $n=4 k+1$ define $\mathbf{A}(n) \in \mathrm{SO}(n)$ and $\mathbf{B}(n-1) \in$ $\mathrm{SO}(n-1)$ by

$$
\mathbf{B}(n-1)=\left[\begin{array}{cc}
0 & \mathbf{I}_{2 k} \\
\mathbf{I}_{2 k} & 0
\end{array}\right], \quad \mathbf{A}(n)=\left[\begin{array}{ll}
1 & \\
& \mathbf{B}(n-1)
\end{array}\right] .
$$

If $n=4 k+2$ define $\mathrm{A}(n) \in \mathrm{SO}(n)$ and $\mathrm{B}(n-1) \in \mathrm{SO}(n-1)$ by

$$
\mathbf{B}(n-1)=\left[\begin{array}{ccc}
1 & & \\
& 0 & \mathbf{I}_{2 k} \\
& \mathbf{I}_{2 k} & 0
\end{array}\right], \quad \mathbf{A}(n)=\left[\begin{array}{ll}
1 & \\
& \mathbf{B}(n-1)
\end{array}\right] .
$$

If $n=4 k$ define $\mathbf{A}(n) \in \mathrm{SO}(n)$ and $\mathbf{B}(n-1) \in \mathrm{SO}(n-1)$ by

$$
\mathbf{B}(n-1)=\left[\begin{array}{ccc}
-1 & & \\
& 0 & \mathbf{I}_{2 k-1} \\
& \mathbf{I}_{2 k-1} & 0
\end{array}\right], \quad \mathbf{A}(n)=\left[\begin{array}{ll}
1 & \\
& \mathbf{B}(n-1)
\end{array}\right] .
$$

The group $\{\mathbf{I}, \mathbf{A}(n)\}$ acts on $\mathrm{SO}(n)$ from the right. Denote the corresponding quotient space by $\mathrm{SO}(n) / \mathbf{A}(n)$. That is $\mathrm{SO}(n) / \mathbf{A}(n)$ is obtained from $\mathrm{SO}(n)$ by the identification $R \sim R \mathbf{A}(n), R \in \mathrm{SO}(n)$.

Proposition 5.2. Let $n=4 k+r, k \geq 1$ and $r=0,1$, 2. Then

$$
\pi_{1}(\mathbf{S O}(n) / \mathbf{A}(n))= \begin{cases}\mathbf{Z}_{4} & \text { if } k \text { is odd } \\ \mathbf{Z}_{2} \oplus \mathbf{Z}_{2} & \text { if } k \text { is even. }\end{cases}
$$

Proof. The exact homotopy sequence of the fibration

$$
\mathrm{Z}_{2} \rightarrow \mathrm{SO}(n) \stackrel{p}{\rightarrow} \mathrm{SO}(n) / \mathrm{A}(n)
$$

gives the exact sequence 


$$
0 \rightarrow \pi_{1}(\mathrm{SO}(n)) \stackrel{p_{*}}{\rightarrow} \pi_{1}(\mathrm{SO}(n) / \mathbf{A}(n)) \rightarrow \pi_{0}\left(\mathbf{Z}_{2}\right) \rightarrow 0 .
$$

Consider a loop $\sigma:[0,1] \rightarrow \mathbf{S O}(n) / \mathbf{A}(n)$ such that $[\sigma] \notin p_{*}\left(\pi_{1}(\mathbf{S O}(n))\right)$. Assume that $\sigma(0)=p(\mathbf{I})$. When lifting $\sigma$ to $\mathbf{S O}(n)$ we obtain a path $\lambda:[0,1] \rightarrow \mathrm{SO}(n)$ such that $\lambda(0)=\mathbf{I}$ and $\lambda(1)=\mathbf{A}(n)$. There are two homotopy classes relative $\partial I$ of such paths. Hence, $\pi_{1}(\operatorname{SO}(n) / \mathbf{A}(n))$ has 4 elements. The loop $\sigma * \sigma$ lifts to $\lambda * \lambda \mathbf{A}(n)$, where $(\lambda \mathbf{A}(n))(t)=\lambda(t) \mathbf{A}(n)$. (Here $*$ denotes path concatenation. Concatenation of two paths is defined if the first one ends where the second one begins.) Now, if

$$
[\lambda * \lambda \mathbf{A}(n)]=\left\{\begin{array}{l}
1 \in \pi_{1}(\mathbf{S O}(n)) \text { then } \pi_{1}(\mathbf{S O}(n) / \mathbf{A}(n)) \cong \mathbf{Z}_{4}, \\
0 \in \pi_{1}(\mathbf{S O}(n)) \text { then } \pi_{1}(\mathbf{S O}(n) / \mathbf{A}(n)) \cong \mathbf{Z}_{2} \oplus \mathbf{Z}_{2} .
\end{array}\right.
$$

We calculate the homotopy class of $\lambda * \lambda \mathbf{A}(n)$ by lifting it to the universal covering $\operatorname{Spin}(n)$ of $\mathrm{SO}(n)$. To this end, note that $\lambda:[0,1] \rightarrow \mathrm{SO}(n)$ lifts to a path

$$
\widetilde{\lambda}:[0,1] \rightarrow \operatorname{Spin}(n) \quad \text { with } \widetilde{\lambda}(0)=+1, \widetilde{\lambda}(1)=\widetilde{\mathbf{A}}(n) .
$$

Hence, the loop $\lambda * \lambda \mathbf{A}(n)$ lifts to the path $\widetilde{\lambda} * \widetilde{\lambda} \widetilde{\mathbf{A}}(n)$, which ends at $\widetilde{\mathbf{A}}(n) \widetilde{\mathbf{A}}(n)$. Now, if

$$
\widetilde{\mathbf{A}}(n) \widetilde{\mathbf{A}}(n)= \begin{cases}+1 & \text { then }[\lambda * \lambda \mathbf{A}(n)]=0 \in \pi_{1}(\mathbf{S O}(n)), \\ -1 & \text { then }[\lambda * \lambda \mathbf{A}(n)]=1 \in \pi_{1}(\mathbf{S O}(n)),\end{cases}
$$

so it remains to compute this product.

Let $\left(e_{1}, \ldots, e_{n}\right)$ denote the standard basis in $\mathrm{R}^{n}$. If $n=4 k+1$ then $\mathbf{A}(n)$ is a composition of reflections in the hyperplanes with unit normals $f_{i}=\frac{1}{\sqrt{2}}\left(e_{i+1}-e_{2 k+i+1}\right)$ and thus

$$
\widetilde{\mathbf{A}}(n)= \pm f_{1} \ldots f_{2 k}, \quad \text { when } n=4 k+1 .
$$

Similarly, we see that

$$
\begin{aligned}
& \widetilde{\mathbf{A}}(n)= \pm f_{1} \ldots f_{2 k}, \quad \text { when } n=4 k+2, \\
& \widetilde{\mathbf{A}}(n)= \pm e_{2} f_{1} \ldots f_{2 k-1}, \quad \text { when } n=4 k,
\end{aligned}
$$

where $f_{i}=\frac{1}{\sqrt{2}}\left(e_{i+2}-e_{2 k+i+2}\right)$. Hence, $\widetilde{\mathbf{A}}(n) \widetilde{\mathbf{A}}(n)$ is, in all the cases, a product of the form $u_{1} \ldots u_{2 k} u_{1} \ldots u_{2 k}$, where the $u_{i}$ are mutually orthonormal unit vectors. It is straightforward to check that

$$
u_{1} \ldots u_{2 k} u_{1} \ldots u_{2 k}=(-1)^{k} \text {. }
$$

5.3. Twist framed circles. Let $c \subset \mathrm{R}^{n}, n=4 k+r, r=0,1,2$, be an embedded circle. Let $p:[0,1] \rightarrow c$ be a parameterization of $c$. Assume that $\mathbf{R}^{n}$ is oriented. 
Definition 5.3. If there is a smooth field of frames $X(p(t))$ in $N\left(c \subset \mathrm{R}^{n}\right)$ such that $X(p(1))=X(p(0)) \mathbf{B}(n-1)$ we say that $c$ is a twist framed circle and denote it by $(c, q, X)$, where $q:[0,1] \rightarrow c$ is given by either $q(t)=p(t)$ or $q(t)=p(1-t)$. The choice being made so that the orientation given by $(\dot{q}, X)$ agrees with that of $\mathrm{R}^{n}$.

Let $(c, q, X)$ be a twist framed circle. Then $(\dot{q}, X)$ gives a framing of $T \mathrm{R}^{n}$ at each point of $c$. Letting the vectors in this frame be column vectors in a matrix the twist framing induces, after orthonormalization, a loop in $\mathrm{SO}(n) / \mathrm{A}(n)$. The homotopy class of this loop will be denoted $[c, q, X]$.

Let $V_{n-1}\left(T \mathrm{R}^{n}\right)$ denote the space of $(n-1)$-frames in $T \mathrm{R}^{n}$.

Definition 5.4. Let $(c, q, X)$ and $(b, p, Y)$ be twist framed circles in $\mathrm{R}^{n}$. We say that these are twist framed isotopic if there is an isotopy

$$
h_{s}:[0,1] \rightarrow V_{n-1}\left(T \mathrm{R}^{n}\right),
$$

such that $h_{0}=(q, X), h_{1}=(p, Y)$ and for each fixed $s$,

$$
\left(\pi h_{s}([0,1]), \pi h_{s}, h_{s}\right)
$$

is a twist framed circle. Here $\pi: V_{n-1}\left(T \mathrm{R}^{n}\right) \rightarrow \mathrm{R}^{n}$ is the projection.

Proposition 5.5. Let $(c, q, X)$ and $(b, p, Y)$ be twist framed circles in $\mathrm{R}^{n}$, $n \geq 4$. Then $(c, q, X)$ and $(b, q, Y)$ are twist framed isotopic if and only if $[c, q, X]=[b, p, Y]$.

Proof. Twist framed isotopy induces homotopy in $\mathrm{SO}(n) / \mathbf{A}(n)$. This proves the only if part. Assume that $[c, q, X]=[b, p, Y]$. Choose a diffeotopy $K_{s}$ of $\mathrm{R}^{n}$ such that $K_{1} q=p$ and $d K_{1}(X(q(0)))=Y(p(0))$. The only if part shows that $\left[b, K_{1} q, d K_{1} X\right]=[b, p, Y]$. Hence, the paths $\left(d K_{1} \dot{q}, d K_{1} X\right)$ and $(\dot{p}, Y)$ in $\mathrm{SO}(n)$ are homotopic with endpoints fixed. It is now easy to complete $\left(K_{s}, d K_{s} X\right)$ to a twist framed isotopy, moving the frame only.

Remark 5.6. The proof of Proposition 5.5 shows that if two twist framed circles are twist framed isotopic then there is an ambient diffeotopy of $\mathrm{R}^{n}$ moving one of them to the other.

5.4. Operations on twist framed circles. Let $(c, q, X)$ be a twist framed circle in $\mathrm{R}^{n}$. Letting $\mathbf{B}(n-1)$ act on the framing we get another twist framed circle $(c, q, X \mathbf{B}(n-1))$. Using Proposition 5.5 we get:

Proposition 5.7. $(c, q, X)$ is twist framed isotopic to $(c, q, X \mathbf{B}(n-1))$.

If $n$ is even then there is another natural operation on twist framed circles in $\mathrm{R}^{n}$. It is associated with reversing the parameterization. 
Definition 5.8. Let $n$ be even. Define $\mathbf{C}(n-1) \in O(n-1)$ and $\mathrm{C}(n) \in \mathrm{SO}(n)$ by

$$
\mathbf{C}(n-1)=\left[\begin{array}{ll}
-1 & \\
& \mathbf{I}_{n-2}
\end{array}\right], \quad \mathbf{C}(n)=\left[\begin{array}{ll}
-1 & \\
& \mathbf{C}(n-1)
\end{array}\right] .
$$

Let $(c, q, X)$ be a twist framed circle in $\mathrm{R}^{n}, n$ even. We can define another twist framed circle $\left(c, q^{\prime}, X^{\prime}\right)$ by

$$
q^{\prime}(t)=q(1-t), \quad X^{\prime}\left(q^{\prime}(t)\right)=X(q(1-t)) \mathbf{C}(n-1) .
$$

Lemma 5.9. If $n=4 k+2$ then $\left[c, q^{\prime}, X^{\prime}\right]=-[c, q, X]$.

Proof. Let $\lambda:[0,1] \rightarrow \mathrm{SO}(n)$ be the path induced by $(c, q, X)$ and $\sigma=p \lambda$ where $p: \mathrm{SO}(n) \rightarrow \mathrm{SO}(n) / \mathrm{A}(n)$. Then the path induced by $\left(c, q^{\prime}, X^{\prime}\right)$ is $\lambda^{-1} \mathbf{C}(n)$. Where $\lambda^{-1}$ denotes $\lambda$ transversed backwards. A uniform rotation through an angle $\pi$ in the plane of the first two vectors in the framing $\left(\dot{q}^{\prime}, X^{\prime}\right)$ gives a homotopy from $p\left(\lambda^{-1} \mathbf{C}(n)\right)$ to $\sigma^{-1}$.

Lemma 5.10. If $n=4 k$ then $\left[c, q^{\prime}, X^{\prime}\right] \neq-[c, q, X]$.

Proof. We have exactly as in the proof of Lemma 5.9 with the same notation as there the paths $\lambda, \lambda^{-1} \mathbf{C}(n):[0,1] \rightarrow \mathbf{S O}(n)$ induced by $(c, q, X)$ and $\left(c, q^{\prime}, X^{\prime}\right)$ respectively. Without loss of generality we may assume that $\lambda(0)=\mathbf{I}$ (and thus $\lambda(1)=\mathbf{A}(n))$. Choose a path $\gamma:[0,1] \rightarrow \mathbf{S O}(n)$, with $\gamma(0)=\mathbf{I}$ and $\gamma(1)=\mathbf{C}(n)$. Then, clearly

$$
p\left(\lambda^{-1} \mathbf{C}(n)\right) \simeq p\left(\gamma \mathbf{A}(n) * \lambda^{-1} \mathbf{C}(n) * \gamma^{-1}\right) .
$$

Note that $\mathbf{C}(n) \mathbf{A}(n)=\mathbf{A}(n) \mathbf{C}(n)$. To prove the lemma it is enough to prove that the loop

$$
\delta=\lambda * \gamma \mathbf{A}(n) * \lambda^{-1} \mathbf{C}(n) * \gamma^{-1},
$$

is nontrivial in $\mathrm{SO}(n)$. Since this implies that

$$
[p \lambda]+\left[p \lambda^{-1} \mathbf{C}(n)\right] \neq 0 \text { in } \pi_{1}(\mathbf{S O}(n) / \mathbf{A}(n)) .
$$

We prove this by lifting $\delta$ to $\operatorname{Spin}(n)$. We have the lifts $\pm \widetilde{\lambda}$ and $\pm \widetilde{\gamma}$ of $\lambda$ and $\gamma$. We assume that $\widetilde{\lambda}(0)=+1=\widetilde{\gamma}(0)$. Then $\widetilde{\lambda}(1)=\widetilde{\mathbf{A}}(n)$ and $\widetilde{\gamma}(1)= \pm e_{1} e_{2}$. Our lift of the loop is the following. Start at +1 , follow $\widetilde{\lambda}$ to $\widetilde{\mathbf{A}}(n)$, follow $\widetilde{\gamma} \widetilde{\mathbf{A}}(n)$ to $\widetilde{\gamma}(1) \widetilde{\mathbf{A}}(n)$. Now, $\widetilde{\mathbf{A}}(n)= \pm e_{2} f_{1} \ldots f_{2 k-1}$ by Proposition 5.2. Hence,

$$
\begin{aligned}
\widetilde{\gamma}(1) \widetilde{\mathbf{A}}(n) & = \pm e_{1} e_{2}\left(e_{2} f_{1} \ldots f_{2 k-1}\right)= \pm f_{1} \ldots f_{2 k-1} e_{1} \\
& = \pm\left(-e_{2} f_{1} \ldots f_{2 k-1} e_{1} e_{2}\right)=-\widetilde{A}(n) \widetilde{\gamma}(1) .
\end{aligned}
$$

So our lifts now follows $-\widetilde{\lambda}^{-1} \widetilde{\gamma}(1)$ until it reaches $-\widetilde{\gamma}(1)$, then it follows $-\widetilde{\gamma}^{-1}$ and ends at -1 . This proves that $\delta$ is nontrival. 
Proposition 5.11. Let $n=4 k+2$ and $(c, q, X)$ be a twist framed circle in $\mathrm{R}^{n}$.

(a) If $k$ is even then $\left(c, q^{\prime}, X^{\prime}\right)$ is twist framed isotopic to $(c, q, X)$.

(b) If $k$ is odd then $\left(c, q^{\prime}, X^{\prime}\right)$ is not twist framed isotopic to $(c, q, X)$.

Proposition 5.12. Let $n=4 k$ and $(c, q, X)$ be a twist framed circle in $\mathrm{R}^{n}$.

(a) If $k$ is even then $\left(c, q^{\prime}, X^{\prime}\right)$ is not twist framed isotopic to $(c, q, X)$.

(b) If $k$ is odd then $\left(c, q^{\prime}, X^{\prime}\right)$ is twist framed isotopic to $(c, q, X)$.

\section{1-dimensional self intersection.}

A generic immersion $f: V^{v} \rightarrow W^{2 v-1}$ has 1-dimensional self intersection. Since any closed connected 1-manifold is a circle, Proposition 2.1 shows that if $V$ is closed then $M_{f}$ is a union of circles. If $V$ and $W$ are orientable and $v$ is even then, by Proposition 2.5, $M_{f}=M_{f}^{\prime \prime}$ (cf. Definition 2.2).

6.1. Definition of the invariants. Let $f: V^{v} \rightarrow W^{2 v-1}$ be a generic immersion. Assume that $V$ and $W$ are spin manifolds with spin structures $\xi_{V}$ and $\xi_{W}$, respectively.Consider a component $c \subset M_{f}^{\prime \prime}$. Proposition 2.10 shows that there is an induced spin structure on $c \approx S^{1}$.

Definition 6.1. If $c \subset M_{f}^{\prime \prime}$ is a component of $M_{f}$, define

$$
s(c)= \begin{cases}1 & \text { if the spin structure induced on } c \text { is trivial, } \\ 0 & \text { if the spin structure induced on } c \text { is the Lie group one. }\end{cases}
$$

REMARK 6.2. We note that

$$
s(c)=\left\langle\xi_{c},[\vec{c}]\right\rangle=\left\langle\left(f^{-1}\right)^{*}\left(\xi_{V}\right),\left[\vec{c}_{V}^{1}\right]\right\rangle+\left\langle\left(f^{-1}\right)^{*}\left(\xi_{V}\right),\left[\vec{c}_{V}^{2}\right]\right\rangle+\left\langle\xi_{W},\left[\vec{c}_{W}\right]\right\rangle,
$$

where $\vec{c}_{V}^{i}, i=1,2$ refer to the extensions of the tangent framing of $c$ to the different sheets of $f(V)$ meeting along $c$ (cf. Remark 2.13).

Lemma 6.3. Let $f: V^{v} \rightarrow W^{2 v-1}$ be a generic immersion. Assume that $V$ and $W$ are spin manifolds. Let $c \subset M_{f}^{\prime \prime}$ be a component of $M_{f}$. If $K_{t}: W \rightarrow W$ and $G_{t}: V \rightarrow V$ are diffeotopies then $K_{1} c$ is a component of $M_{K_{1} f G_{1}}^{\prime \prime}$ and $s(c)=s\left(K_{1} c\right)$.

Proof. Let $b=K_{1} c$. We need to compute

$$
\begin{aligned}
s(b) & =\left\langle\xi_{b},[\vec{b}]\right\rangle=\left\langle\left(\left(K_{1} f G_{1}\right)^{-1}\right)^{*}\left(\xi_{V}\right),\left[\vec{b}_{V}^{1}\right]\right\rangle \\
& +\left\langle\left(\left(K_{1} f G_{1}\right)^{-1}\right)^{*}\left(\xi_{V}\right),\left[\vec{b}_{V}^{2}\right]\right\rangle+\left\langle\xi_{W},\left[\vec{b}_{W}\right]\right\rangle,
\end{aligned}
$$

Remark 2.12 allows us to take the extensions of the framing of $b$ as those induced by $d K_{1}$ acting on the extensions of the framingof $c$. We have, for $i=1,2$, 


$$
\begin{aligned}
\left\langle\left(\left(K_{1} f G_{1}\right)^{-1}\right)^{*}\left(\xi_{V}\right),\left[\vec{b}_{V}^{i}\right]\right\rangle & =\left\langle\left(K_{1}^{-1}\right)^{*}\left(f^{-1}\right)^{*}\left(G_{1}^{-1}\right)^{*}\left(\xi_{V}\right),\left[\vec{b}_{V}^{i}\right]\right\rangle \\
& =\left\langle\left(K_{1}^{-1}\right)^{*}\left(f^{-1}\right)^{*}\left(\xi_{V}\right),\left[\vec{b}_{V}^{i}\right]\right\rangle \\
& =\left\langle\left(f^{-1}\right)^{*}\left(\xi_{V}\right),\left(K_{1}^{-1}\right)_{*}\left(K_{1}\right)_{*}\left[\vec{c}_{V}^{i}\right]\right\rangle \\
& =\left\langle\left(f^{-1}\right)^{*}(\xi V),\left[\vec{c}_{V}^{i}\right]\right\rangle,
\end{aligned}
$$

where the second equality follows from the fact that $d G_{1}$ is homotopic to identity. Also,

$$
\left\langle\xi_{W},\left(K_{1}\right)_{*}\left[\vec{c}_{W}\right]\right\rangle=\left\langle\xi_{W},\left[\vec{c}_{W}\right]\right\rangle,
$$

since $d K_{1}$ is homotopic to the identity. It follows that $s(b)=s(c)$.

When describing the invariants associated to nontrivially covered components we restrict ourselves to the case when the target manifold is Euclidean space. Let $f: V^{v} \rightarrow \mathrm{R}^{2 v-1}$ be a generic immersion. Assume that $v$ is odd, $\mathrm{R}^{2 v-1}$ is oriented and that $V$ is equipped with a spin structure $\xi_{V}$. We shall construct a functional $\omega_{f}$ on components of $M_{f}^{\prime}$ which takes values in $\pi_{1}(\mathrm{SO}(2 v-1) / \mathbf{A}(2 v-1))$. Let $c \subset M_{f}^{\prime}$ be a component of $M_{f}$. Since $v-1$ is even we know by Proposition that there is an induced orientation of $c$. Let $f^{-1}(c)=c_{V}$. Choose a parameterization $q:[0,1] \rightarrow c$ of $c$, such that the orientation given by this parameterization agrees with that induced on $c$. Then $q$ lifts to two paths $q_{i}:[0,1] \rightarrow V$ such that $q_{1}(0)=q_{2}(1), q_{1}(1)=q_{2}(0)$ and $q_{1} * q_{2}$ is a parameterization of $c_{V}$. Choose a framing $Y$ of $N\left(c_{V} \subset V\right)$ such that, completing $Y$ with the tangent vector of $c_{V}$ coming from the parameterization $q_{1} * q_{2}$ we get a framing of $T V \mid c_{V}$, oriented coherently with the orientation of $V$. We denote the framed curve obtained in this way by $\vec{c}_{V}$. Let

$$
X(q(t))=\left(d f Y\left(q_{1}(t)\right), d f Y\left(q_{2}(t)\right)\right) .
$$

Then $(c, q, X)$ is a twist framed circle.

Let $u \in \pi_{1}(\mathrm{SO}(n)), n \geq 3$, denote the nontrivial element. If $p: \mathrm{SO}(n) \rightarrow$ $\mathrm{SO}(n) / \mathbf{A}(n)$ then $p_{*}(u) \in \pi_{1}(\mathrm{SO}(n) / \mathbf{A}(n))$ is an element of order 2. For $x \in \mathbf{Z}_{2}$ let $x \cdot p_{*}(u)$ denote the value at $x$ of the homomorphism $\mathbf{Z}_{2} \rightarrow \pi_{1}(\mathrm{SO}(n) / \mathbf{A}(n))$ taking 1 to $p_{*}(u)$.

\section{Definition 6.4.}

$$
\omega_{f}(c)=\left\langle\xi_{V},\left[\vec{c}_{V}\right]\right\rangle \cdot p_{*}(u)+[c, q, X] \in \pi_{1}(\mathrm{SO}(2 v-1) / \mathbf{A}(2 v-1))
$$

Lemma 6.5. The value of $\omega_{f}(c)$ does neither depend on the choice of parameterization nor on the choice of normal framing of $c_{V}$.

Proof. Let $q^{\prime}$ and $q$ be two parameterizations. Since their orientations must agree they are homotopic. Hence also the induced parameterizations of 
$c_{V}$ are homotopic so neither of the terms on the right hand side in the definition of $\omega_{f}(c)$ change. Let $Y_{1}$ and $Y_{2}$ be framings of $N(c \subset V)$ giving rise to framed curves $\vec{c}_{V}^{1}$ and $\vec{c}_{V}^{2}$ respectively. These are framed isotopic, moving the frame only, if and only if

$$
\left\langle\xi_{V},\left[\vec{c}_{V}^{1}\right]\right\rangle=\left\langle\xi_{V},\left[\vec{c}_{V}^{2}\right]\right\rangle
$$

Such a framed isotopy clearly gives rise to a twist framed isotopy between $\left(c, q, X_{1}\right)$ and $\left(c, q, X_{2}\right)$ where $X_{i}$ is the framing induced by $d f$ acting on $Y_{i}$. Hence,

$$
\left\langle\xi_{V},\left[\vec{c}_{V}^{1}\right]\right\rangle=\left\langle\xi_{V},\left[\vec{c}_{V}^{2}\right]\right\rangle \text { implies }\left[c, q, X_{1}\right]=\left[c, q, X_{2}\right]
$$

by Proposition 5.5. Now, if

$$
\left\langle\xi_{V},\left[\vec{c}_{V}^{1}\right]\right\rangle \neq\left\langle\xi_{V},\left[\vec{c}_{V}^{2}\right]\right\rangle
$$

then we can change the framing $Y_{2}$ by a locally supported rotation of the frame, such that $\left\langle\xi_{V},\left[\vec{c}_{V}^{2}\right]\right\rangle$ changes by 1 and $\left[c, q, X_{2}\right]$ by addition of $p_{*}(u)$.

Lemma 6.6. Let $K_{t}: \mathrm{R}^{2 v-1} \rightarrow \mathrm{R}^{2 v-1}$ and $G_{t}: V \rightarrow V$ be diffeotopies. Then $K_{1} c \subset M_{K_{1} f G_{1}}^{\prime}$ is a component of $M_{K_{1} f G_{1}}$ and $\omega_{K_{1} f G_{1}}\left(K_{1} c\right)=\omega_{f}(c)$.

Proof. It follows from Lemma 6.5 that we can assume that the twist framing on $K_{1} c$ is induced from that of $c$ by appropriate actions of diffeotopies. That the first term on the right hand side in the definition of $\omega_{f}(c)$ does not change follows from the fact that $G_{t}$ is a diffeotopy. That the second does not change follows from the fact that the diffeotopies gives rise to a twist framed isotopy and Proposition 5.5.

For $n=4 k$ fix isomorphisms

$$
\phi: \pi_{1}(\mathbf{S O}(n) / \mathbf{A}(n)) \rightarrow \begin{cases}\mathbf{Z}_{4} & \text { if } k \text { is odd } \\ \mathbf{Z}_{2} \oplus \mathbf{Z}_{2} & \text { if } k \text { is even, }\end{cases}
$$

such that $\phi\left(p_{*}(u)\right)=2$, when $k$ is odd and $\phi\left(p_{*}(u)\right)=(1,1)$, when $k$ is even.

DEFINITION 6.7.

(a) If $v \equiv 1 \bmod 4$ and $c \subset M_{f}^{\prime}$ is a component of $M_{f}$, define

$$
s(c)= \begin{cases}1 & \text { if } \phi\left(\omega_{f}(c)\right)=(1,0), \\ 0 & \text { if } \phi\left(\omega_{f}(c)\right)=(0,1) .\end{cases}
$$

(b) If $v \equiv 3 \bmod 4$ and $c \subset M_{f}^{\prime}$ is a component of $M_{f}$, define

$$
s(c)= \begin{cases}1 & \text { if } \phi\left(\omega_{f}(c)\right)=1 \\ 0 & \text { if } \phi\left(\omega_{f}(c)\right)=3\end{cases}
$$


We now turn to the general case: Let $f: V^{v} \rightarrow \mathrm{R}^{2 v-1}$ be a generic immersion. Assume that $V^{v}$ is equipped with a spin structure and that $\mathrm{R}^{2 v-1}$ is oriented. Let $c$ be a component of $M_{f}$.

Lemma 6.8. Let $K_{t}: \mathrm{R}^{2 v-1} \rightarrow \mathrm{R}^{2 v-1}$ and $G_{t}: V \rightarrow V$ be diffeotopies. Then $K_{1} c$ is a component of $M_{K_{1} f G_{1}}, K_{1} c \subset M_{K_{1} f G_{1}}^{\prime}$ if and only if $c \subset M_{f}^{\prime}$ and $s(c)=s\left(K_{1} c\right)$.

Proof. This follows from Lemmas 6.3, 6.6.

6.2. Proof of Theorem 1.4. The assumption, in Theorem 1.4, that $V$ is $2-$ connected implies that there is a unique spin structure on $V$. Hence, $s$ is well defined if we fix orientations on $V$ and $\mathrm{R}^{2 v-1}$. We assume in the proof below that this is done.

The only if part follows from the fact that the diffeomorphism type of the self intersection is preserved under diffeotopy equivalence and Lemma 6.8. Assume that $c$ and $b$ are components of $M_{f}$ and $M_{h}$ respectively. Let $f^{-1}(c)=\widetilde{c}, h^{-1}(b)=\widetilde{b}$ and assume that $\widetilde{c}$ and $\widetilde{b}$ have the same number of components. Choose diffeotopies $K_{\widetilde{b}}^{\prime}: \mathrm{R}^{2 v-1} \rightarrow \mathrm{R}^{2 v-1}$ and $G_{t}: V \rightarrow V$ such that $K_{1}^{\prime} c=b, G_{1} \widetilde{b}=\widetilde{c}, K_{1}^{\prime} f G_{1}|\widetilde{b}=h| \widetilde{b}$ and such that the induced orientations agree. By Lemma 2.14 we can, up to diffeotopy equivalence, locally replace $K_{1}^{\prime} f G_{1}=f^{\prime}$ and $h$ by their normal derivatives on $N(\widetilde{b} \subset V)$.

First, suppose that $c \subset M_{f}^{\prime \prime}, b \subset M_{h}^{\prime \prime}$ and $s(c)=s(b)$. Write $\widetilde{b}=b_{1} \cup b_{2}$, where $b_{1}$ and $b_{2}$ are the components of $\widetilde{b}$. Pick a framing of $N\left(b_{1} \cup b_{2} \subset V\right)$. Then $f^{\prime}$ and $h$ induce framings of $N\left(b \subset \mathrm{R}^{2 v-1}\right)$. Consider the formula in Remark 6.2, associated to $f^{\prime}$ and $h$ respectively. Since $s(b)=s(c)$ Lemma 6.3 tells us that the terms $\left\langle\xi_{b},[\vec{b}]\right\rangle$, associated to $f^{\prime}$ and $h$ respectively, agree. Moreover,

$$
\left\langle\left(f^{\prime-1}\right)^{*} \xi_{V},\left[d f^{\prime}\left(\vec{b}_{V}^{i}\right)\right]\right\rangle=\left\langle\xi_{V},\left[\vec{b}_{V}^{i}\right]\right\rangle=\left\langle\left(h^{-1}\right)^{*} \xi_{V},\left[d h\left(\vec{b}_{V}^{i}\right)\right]\right\rangle, \text { for } i=1,2 .
$$

Hence, the terms of the form $\left\langle\xi_{\mathrm{R}^{2 v-1}},\left[\vec{b}_{\mathrm{R}^{2 v-1}}\right]\right\rangle$ agree as well. But this means that there is a diffeotopy of $\mathrm{R}^{2 v-1}$ fixed on $b$ and supported in a neighborhood of $b$ taking the framing induced by $f^{\prime}$ to that induced by $h$.

Secondly, suppose that $c \subset M_{f}^{\prime}, b \subset M_{h}^{\prime}$ and $s(c)=s(b)$. Pick a framing of $N(\widetilde{b} \subset V)$. Then $f^{\prime}$ and $h$ induce twist framings of $N\left(b \subset \mathrm{R}^{2 v-1}\right)$. Consider the formula in Definition 6.4, associated to $f^{\prime}$ and $h$ respectively. Since $s(c)=s(b)$, Lemma 6.6 tells us that $\omega_{f^{\prime}}(b)=\omega_{h}(b)$. Note that the terms $\left\langle\xi_{V},\left[\vec{b}_{V}\right]\right\rangle$ are independent of the immersions. Hence, the terms of the form $[b, q, X]$, associated to $f^{\prime}$ and $h$ respectively, must agree. Then, by Remark 5.6 , there is a diffeotopy of $\mathrm{R}^{2 v-1}$ fixed on $b$ taking the twist framing induced by $f^{\prime}$ to that induced by $h$. Repeating the above construction for all components of $M_{h}$ we find diffeotopies $K_{t}: \mathrm{R}^{2 v-1} \rightarrow \mathrm{R}^{2 v-1}$ and $L_{t}: V \rightarrow V$ such that 
$K_{1} f L_{1}|W=h| W$, where $W$ is a neighborhood of $\widetilde{M}_{h}$. An application of Theorem 1.2 finishes the proof.

\section{2-dimensional self intersection.}

7.1. The first two invariants. Let $f: V^{v} \rightarrow W^{2 v-2}$ be a generic immersion. If $V$ is closed, Proposition 2.1 shows that $M_{f}$ is a closed surface. Assume that $V$ and $W$ are orientable and let $F$ be a component of $M_{f}$. The topological type of both $F$ and $f^{-1}(F)=\widetilde{F}$ is determined by the genus $g(F)$ of $F$ and the number of components of $\widetilde{F}$ : Denote the surface obtained by connected sum of $k$ tori by $\Sigma_{k}$ and that obtained by connected sum of $k$ projective planes by $\Gamma_{k}$. Let $g=g(F)$. Proposition 2.1 gives:

$$
F \subset M_{f}^{\prime \prime} \text { implies } F \approx \Sigma_{g} \text { and } \widetilde{F} \approx \Sigma_{g} \cup \Sigma_{g} .
$$

Propositions 2.3, 2.5 give:

(a) If $v$ is even then $F \subset M_{f}^{\prime}$ implies $F \approx \Sigma_{g}$ and $\widetilde{F} \approx \Sigma_{2 g-1}$.

(b) If $v$ is odd then $F \subset M_{f}^{\prime}$ implies $F \approx \Gamma_{g}$ and $\widetilde{F} \approx \Sigma_{g-1}$.

7.2. Disconnected double locus and spin structure. A spin structure $\xi$ on a surface $F$ induces a quadratic function $q_{\xi}: H_{1}\left(F ; Z_{2}\right) \rightarrow Z_{2}$, that is, if $a, b \in H_{1}\left(F ; Z_{2}\right)$ then $q_{\xi}(a+b)=q_{\xi}(a)+q_{\xi}(b)+a \cdot b$, where $a \cdot b$ denotes the intersection pairing. Let $z \in H_{1}\left(\mathrm{SO}(T F) ; Z_{2}\right)$ denote the fiber class. Let $a \in H_{1}\left(F ; Z_{2}\right)$. Then we can compute $q_{\xi}(a)$ as follows. Pick a simple closed curve $\alpha$ in $F$ such that $[\alpha]=a$, where $[\alpha]$ denotes the $Z_{2}$-homology class of $\alpha$. Let $\vec{\alpha}$ denote $\alpha$ framed with its unit tangent vector, an orientation of $F$ picks a normal vector to complete it to a framing of $T F$ along $\alpha$. We get the element $[\vec{\alpha}] \in H_{1}\left(\mathrm{SO}(T F) ; \mathbf{Z}_{2}\right)$ and

$$
q_{\xi}(a)=\langle\xi,[\vec{\alpha}]+z\rangle,
$$

see [7] for reference. Let $\left(L^{2 g}, \cdot\right)$ be a symplectic vector space over $Z_{2}$. Let $q: L \rightarrow Z_{2}$ be a quadratic function on $L$. The Arf invariant of $q$ is defined by

$$
\operatorname{Arf}(q)=\sum_{i} q\left(a_{i}\right) q\left(b_{i}\right)
$$

where $\left\{a_{i}, b_{i}\right\}_{i=1}^{g}$ is a symplectic basis of $L$. If $q_{1}$ and $q_{2}$ are quadratic functions on $L$ then there is a symplectomorphism $h: L \rightarrow L$ such that $q_{2}=q_{1} h$ if and only if $\operatorname{Arf}\left(q_{1}\right)=\operatorname{Arf}\left(q_{2}\right)$.

Let $f: V^{v} \rightarrow W^{2 v-2}$ be a generic immersion and let $F \subset M_{f}^{\prime \prime}$ be a component of $M_{f}$. Assume that there are spin structures $\xi_{V}$ and $\xi_{W}$ on $V$ and $W$ respectively. By Proposition 2.10, there is an induced spin structure $\xi_{F}$ on $F$. The quadratic function on $H_{1}\left(F ; Z_{2}\right)$ induced by $\xi_{F}$ will be denoted $q_{f, F}$, where $f$ refers to the map. 
Definition 7.1. If $F \subset M_{f}^{\prime \prime}$, define $s(F)=\operatorname{Arf}\left(q_{f, F}\right) \in \mathrm{Z}_{2}$.

Lemma 7.2. Let $c$ be a simple closed curve in $F \subset M_{f}^{\prime \prime}$. If $K_{t}: W \rightarrow W$ and $G_{t}: V \rightarrow V$ are diffeotopies then $K_{1} c$ is a simple closed curve in $K_{1} F$, which is a component of $M_{K_{1} f G_{1}}^{\prime \prime}$ and

$$
q_{K_{1} f G_{1}, K_{1} F}\left(\left[K_{1} c\right]\right)=q_{f, F}([c]) .
$$

Proof. By formula $(\dagger)$ above it is enough to check that

$$
\left\langle\xi_{K_{1} F},\left[K_{1} \vec{c}\right]\right\rangle=\left\langle\xi_{F},[\vec{c}]\right\rangle,
$$

this follows from the calculation in the proof of Lemma 6.3.

Lemma 7.3. Let $f, h: V^{v} \rightarrow W^{2 v-2}$ be two diffeotopy equivalent generic immersions. Let $F$ be the component of $M_{f}$ corresponding, under the diffeotopy equivalence, to the component $G$ of $M_{h}$ then $g(F)=g(G)$. Assume that $F \subset M_{f}^{\prime \prime}$, then $G \subset M_{h}^{\prime \prime}$ and $s(F)=s(G)$.

Proof. This is a direct consequence of Lemma 7.2 and the fact that if $\left\{a_{i}, b_{i}\right\}$ is a symplectic basis for $H_{1}\left(F ; Z_{2}\right)$ then $\left\{K_{1} a_{i}, K_{1} b_{i}\right\}$ is a symplectic basis for $H_{1}\left(K_{1} F ; Z_{2}\right)$.

7.3. Connected double locus and a functional on curves. We restrict ourselves to the case when the target manifold is Euclidean space. Let $f: V^{v} \rightarrow \mathrm{R}^{2 v-2}$ be a generic immersion. Let $F$ be a componentof $M_{f}^{\prime}$. Assume that $V$ is equipped with a spin structure $\xi_{V}$ and that $\mathrm{R}^{2 v-2}$ is oriented. We shall construct a functional $\omega_{f}$ on (oriented) immersed, closed curves in $F$, which takes values in $\pi_{1}(\mathrm{SO}(2 v-2) / \mathbf{A}(2 v-2))$.

Let $u \in \pi_{1}(\mathrm{SO}(n))$ denote the nontrivial element. Let $p: \mathrm{SO}(n) \rightarrow \mathrm{SO}(n) /$ $\mathbf{A}(n)$ be the quotient map. Then $p_{*}(u) \in \pi_{1}(\mathrm{SO}(n) / \mathbf{A}(n))$ is an element of order 2.

Let $c \subset F$ be an immersed curve whose only self intersectionsare transverse double points. The inverse image $f^{-1}(c)=\widetilde{c}$ may be either a connected or a disconnected subset of $f^{-1}(F)=\widetilde{F}$. Choose a parameterization $r:[0,1] \rightarrow c$.

(a) When $\widetilde{c}$ is disconnected $r$ lifts to two parameterizations $r_{i}:[0,1] \rightarrow c_{i}$, $i=1,2$, where $c_{1}$ and $c_{2}$ are disjoint immersed closed curves in $\widetilde{F}$. Choose framings $Y_{i}$ of $N(\widetilde{F} \subset V) \mid c_{i}$. Adding the tangent $\dot{r}_{i}$ and the normal $n_{i}$ to $Y_{i}$, the normal chosen so that $\left(\dot{r}_{i}, n_{i}, Y_{i}\right)$ is positively oriented, we get two framed curves $\vec{c}_{i}, i=1,2$. Let $\left[\vec{c}_{V}\right]$ denote the homology class in $H_{1}\left(\mathrm{SO}(T V) ; \mathrm{Z}_{2}\right)$ given by

$$
\left[\vec{c}_{V}\right]=\left[\vec{c}_{1}\right]+\left[\vec{c}_{2}\right]
$$


where $\left[\vec{c}_{i}\right] \in H_{1}\left(\mathrm{SO}(T V) ; \mathrm{Z}_{2}\right)$ is obtained as usual, by viewing a framed curve as a curve in the principal bundle. Now, $f$ induces the framing

$$
X(p(t))=\left(\dot{r}, n(r(t)), d f Y_{1}\left(r_{1}(t)\right), d f Y_{2}\left(r_{2}(t)\right)\right),
$$

of $T \mathrm{R}^{2 v-2} \mid c$, where $n$ is chosen so that the framing gives the orientation of $\mathrm{R}^{2 v-2}$. This framing induces a loop in $\mathrm{SO}(2 v-2)$, composing with the quotient map $p: \mathrm{SO}(2 v-2) \rightarrow \mathrm{SO}(2 v-2) / \mathbf{A}(2 v-2)$ we get an element $[c, r, X] \in \pi_{1}(\mathrm{SO}(2 v-2) / \mathbf{A}(2 v-2))$.

(b) When $\widetilde{c}$ is connected, $r$ lifts to $r_{i}:[0,1] \rightarrow \widetilde{F}, i=1,2$ such that $r_{1}(0)=r_{2}(1), r_{1}(1)=r_{2}(0)$ and $r_{1} * r_{2}$ is a parameterization of $\widetilde{c}$. Choose a framing $Y$ of $N(\widetilde{F} \subset V) \mid \widetilde{c}$. Adding the tangent vector given by the parameterization and the appropriate normal vector we get a framing of $T V \mid \widetilde{c}$. This framed curve gives an element $\left[\vec{c}_{V}\right] \in H_{1}\left(\mathrm{SO}(T V) ; \mathrm{Z}_{2}\right)$. Now, $f$ induces the twist framing

$$
X(p(t))=\left(\dot{r}, n(r(t)), d f Y_{1}\left(r_{1}(t)\right), d f Y_{2}\left(r_{2}(t)\right)\right),
$$

of $c$, where $n$ is an appropriately chosen normal vector. As in Section 5.3 we get $[c, r, X] \in \pi_{1}(\mathrm{SO}(2 v-2) / \mathbf{A}(2 v-2))$.

REMARK 7.4. If the framed curve constructed in (a) above is denoted $\vec{c}$ and $\xi$ denotes the unique spin structure on $\mathrm{R}^{2 v-2}$ then $[c, r, X]=\langle\xi,[\vec{c}]\rangle \cdot p_{*}(u)$.

DeFinition 7.5.

$$
\omega_{f}(c, r)=\left\langle\xi_{V},\left[\vec{c}_{V}\right]\right\rangle \cdot p_{*}(u)+[c, r, X]+p_{*}(u) \in \pi_{1}(\operatorname{SO}(2 v-2) / \mathbf{A}(2 v-2)) .
$$

Lемма 7.6. The value of $\omega_{f}(c, r)$ does neither depend on the choice of framing of $N(\widetilde{F} \subset V) \mid \widetilde{c}$ nor on the numbering of the liftings of $r$.

Proof. The first statement follows in the case of induced twist framing exactly as the corresponding statement in Lemma 6.5. In the case of framing, see Remark 2.12. The second statement follows immediately when there is an induced framing. When there is an induced twist framing it follows from Proposition 5.7.

Lemma 7.7. Let $f: V^{v} \rightarrow \mathrm{R}^{2 v-2}$ be a generic immersion. Let $F \subset M_{f}^{\prime}$ be a component and let $c \subset F$ be a closed curve parameterized by $r$. If $K_{t}: \mathrm{R}^{2 v-2} \rightarrow \mathrm{R}^{2 v-2}$ and $G_{t}: V \rightarrow V$ are diffeotopies then $K_{1} F$ is a component of $M_{K_{1} f G_{1}}^{\prime}, K_{1} c \subset K_{1} F$ is a closed curve with parameterization $K_{1} r$ and

$$
\omega_{K_{1} f G_{1}}\left(K_{1} c, K_{1} r\right)=\omega_{f}(c, r)
$$

Proof. Let $K_{1} c=b$. By Lemma 7.6, we can take the framing of $\widetilde{b}$ to be induced by $G_{1}$ acting on the framing of $\widetilde{c}$ and similarly for the framing or twist framing induced on $b$. Then $\left[\vec{b}_{V}\right]=\left[\vec{c}_{V}\right]$, since the curve or curves are 
framed isotopic. If $Y$ denotes the framing or twist framing of $b$ then $[c, r, X]=\left[b, K_{1} r, Y\right]$ for the same reason.

Lemma 7.8. If $r$ and $r^{\prime}$ are homotopic parameterizations of $c$ then

$$
\omega_{f}(c, r)=\omega_{f}\left(c, r^{\prime}\right) .
$$

Proof. An homotopy between $r$ and $r^{\prime}$ induces homotopies everywhere in the construction preceding Definition 7.5.

There are two nonhomotopic parameterizations of a curve as above. If $r:[0,1] \rightarrow c$ is a parameterization and $r^{\prime}(t)=r(1-t)$ then $r$ and $r^{\prime}$ represents different homotopy classes.

Lemma 7.9. Let $c, r$ and $r^{\prime}$ be as described above. Let $v$ denote the dimension of the source space of the generic immersion $f$.

(a) If $v \equiv 0 \bmod 4$ or $v \equiv 1 \bmod 4$ and $\widetilde{c}$ is connected then

$$
\omega_{f}\left(c, r^{\prime}\right)=\omega_{f}(c, r)+p_{*}(u) .
$$

(b) If $v \equiv 2 \bmod 4$ or $v \equiv 3 \bmod 4$ or $\widetilde{c}$ is disconnected then

$$
\omega_{f}\left(c, r^{\prime}\right)=\omega_{f}(c, r) \text {. }
$$

Proof. This is follows from Lemmas 5.9, 5.10 and Remark 7.4.

Because of Lemmas 7.8, 7.9, we write $\omega_{f}(c)$, dropping the parameterization, and regard $c$ as an immersed curve, if $v \equiv 2 \bmod 4$ or $v \equiv 3 \bmod 4$, or as an oriented immersed curve, if $v \equiv 0 \bmod 4$ or $v \equiv 1 \bmod 4$.

Lemma 7.10. Let $c \subset F$ be a curve that bounds a disk in $F$ then $\omega_{f}(c)=0$.

Proof. Since $c$ bounds a disk $D, \widetilde{c}$ is disconnected and moreover, $D$ is the intersection of two disjoint sheets of $f(V)$. Hence, there is an induced spin structure on $D$, and the spin structure on $c$ must be the trivial one. By Remark 2.13 this means that

$$
\left\langle\xi_{D},\left[\vec{c}_{D}\right]\right\rangle=1 .
$$

Clearly, we can choose the extensions of the framing so that

$$
\left\langle\xi_{V},\left[\vec{c}_{V}\right]\right\rangle=0,
$$

where $\vec{c}_{V}$ is obtained by pulling back the extended framings via $f$. Hence, we must have

$$
\left\langle\xi_{\mathrm{R}^{2 v-2}},\left[\vec{c}_{\mathrm{R}^{2 v-2}}\right]\right\rangle=1,
$$


but this means that $[c, r, X]=p_{*}(u)$ for any parameterization $r$. We get

$$
\omega_{f}(c)=0+p_{*}(u)+p_{*}(u)=0 .
$$

LEMMA 7.11. If $b$ and $c$ are isotopic embedded curves in $F$ then $\omega_{f}(b)=$ $\omega_{f}(c)$.

Proof. The isotopy between $b$ and $c$ extends to an isotopy of $F$ and then to an isotopy of $\mathrm{R}^{2 v-2}$. Also, the isotopy, of $F$ lifts and the lifted isotopy extends to an isotopy of $V$. The lemma now reduces to Lemma 7.7.

Let $c$ be an immersed closed (oriented) curve in $F$ with at least one double point. Smoothing $c$ at this double point, we obtain two immersed, closed (oriented) curves $a$ and $b$ in $F$.

LEMMA 7.12. $\omega_{f}(c)=\omega_{f}(a)+\omega_{f}(b)+p_{*}(u)$.

Proof. Let $x \in F$ be the crossing point. The smoothing takes place in a small disk $D$ about $x$. Now, $f^{-1}(D)=D_{1} \cup D_{2}$ and we can assume that the framings $Y$ of $N(\widetilde{F} \subset V) \mid \widetilde{c}$ is chosen to be constant over $D_{i}$. The smoothing lifts to two smoothings in $D_{1}$ and $D_{2}$. If we let the framing of $\widetilde{a}$ and $\widetilde{b}$ be that induced by the framing of $\widetilde{c}$ just changing the part in $T \widetilde{F}$ over $D_{i}, i=1,2$. Then (cf. [7])

$$
\left[\vec{c}_{V}\right]=\left[\vec{a}_{V}\right]+\left[\vec{b}_{V}\right]
$$

We next consider the situation in $T \mathrm{R}^{2 v-2} \mid F$. We choose the parameterization $r$ of $c$ so that it starts and ends at $x$. Let $r_{1}$ and $r_{2}$ denote the induced parameterizations and $X_{1}$ and $X_{2}$ the induced (twist) framings of $a$ and $b$ respectively. Let $\lambda(a), \lambda(b)$ and $\lambda(c)$ be the paths in $\mathrm{SO}(n)$ induced by the framings or twist framings of $a, b$ and $c$ respectively. We treat the different cases:

1. If $a$ and $b$ are both twist framed then $c$ is framed and

$$
\lambda(c) \simeq \lambda(a) * \lambda(b) \mathbf{A}(2 v-2) .
$$

2. If $a$ is twist framed and $b$ is framed then $c$ is twist framed and

$$
\lambda(c) \simeq \lambda(a) * \lambda(b) \mathbf{A}(2 v-2) .
$$

3. If $a$ and $b$ are both framed then $c$ is framed and

$$
\lambda(c) \simeq \lambda(a) * \lambda(b) .
$$

Since $[c, r, X]=[p(\lambda(c))]$ and similarly for $a$ and $b$ we see that,in all the cases above, we have

$$
[c, r, X]=\left[a, r_{1}, X_{1}\right]+\left[b, r_{2}, X_{2}\right]
$$

Finally, 


$$
\begin{aligned}
\omega_{f}(c) & =\left\langle\xi_{V},\left[\vec{c}_{V}\right]\right\rangle \cdot p_{*}(u)+[c, r, X]+p_{*}(u) \\
& =\left\langle\xi_{V},\left[\vec{a}_{V}\right]+\left[\vec{b}_{V}\right]\right\rangle \cdot p_{*}(u)+\left[a, r_{1}, X_{1}\right]+\left[b, r_{2}, X_{2}\right]+p_{*}(u) \\
& =\left\langle\xi_{V},\left[\vec{a}_{V}\right]\right\rangle \cdot p_{*}(u)+\left[a, r_{1}, X_{1}\right] \\
& +\left\langle\xi_{V},\left[\vec{b}_{V}\right]\right\rangle \cdot p_{*}(u)+\left[b, r_{1}, X_{1}\right]+p_{*}(u)=\omega_{f}(a)+\omega_{f}(b)+p_{*}(u) .
\end{aligned}
$$

Let $a$ and $b$ be closed immersed (oriented) curves in $F$ that intersect transversely. By smoothing at one of the intersection points we get a closed immersed (oriented) curve $c$.

Lemma 7.13. $\omega_{f}(c)=\omega_{f}(a)+\omega_{f}(b)+p_{*}(u)$.

Proof. The proof is similar to the proof of Lemma 7.12.

We next extend the function $\omega_{f}$.

Definition 7.14. Let $L=c_{1} \cup \ldots \cup c_{k}$ be a disjoint union of embedded (oriented) circles in $F$, define

$$
\omega_{f}(L)=\sum_{i} \omega_{f}\left(c_{i}\right)
$$

Lemma 7.15. Let $L_{1}$ and $L_{2}$ be two unions of disjoint embedded (oriented) circles in $F$, intersecting transversely in $k$ points. Then we get a new collection of (oriented) embedded circles $L_{3}$ by smoothing at these points and

$$
\omega_{f}\left(L_{3}\right)=\omega_{f}\left(L_{1}\right)+\omega_{f}\left(L_{2}\right)+k \cdot p_{*}(u) .
$$

Where $k \cdot p_{*}(u)$ denotes the value at $k$ of the homomorphism $\mathrm{Z} \rightarrow \pi_{1}(\mathrm{SO}(n) / \mathbf{A}(n))$ taking 1 to $p_{*}(u)$.

Proof. Induction using Lemmas 7.12, 7.13.

For $n=4 k+r, r=0,2$ choose isomorphisms

$$
\phi: \pi_{1}(\mathbf{S O}(n) / \mathbf{A}(n)) \rightarrow \begin{cases}\mathbf{Z}_{4} & \text { if } k \text { is odd } \\ \mathbf{Z}_{2} \oplus \mathbf{Z}_{2} & \text { if } k \text { is even, }\end{cases}
$$

such that $\phi\left(p_{*}(u)\right)=2$, when $k$ is odd, and $\phi\left(p_{*}(u)\right)=(1,1)$, when $k$ is even.

Definition 7.16. Let $f: V^{v} \rightarrow \mathrm{R}^{2 v-2}$ be a generic immersion. Assume that $V$ is equipped with a spin structure and that $\mathrm{R}^{2 v-2}$ is oriented. Let $F \subset M_{f}^{\prime}$ be a component of $M_{f}$. Let $L$ be a disjoint union of circles in $F$. (If $v \equiv r \bmod 4$, $r=0,1$ we assume that the circles are also oriented.) Define

$$
\hat{q}_{f}(L)=\phi\left(\omega_{f}(L)\right) .
$$

We list the properties of $\hat{q}_{f}$ that follow from the results above in the following proposition. 
Proposition 7.17.

(a) If $L_{1}$ and $L_{2}$ are disjoint unions of (oriented) circles in $F$ that intersect in $k$ points then we can get adisjoint union $L_{3}$ of (oriented) circles by smoothing all intersections and

$$
\hat{q}_{f}\left(L_{3}\right)=\hat{q}_{f}\left(L_{1}\right)+\hat{q}_{f}\left(L_{2}\right)+k \cdot\left\{\begin{array}{c}
2 \\
(1,1)
\end{array}\right\},
$$

which one of 2 and $(1,1)$ we use depends on the dimension.

(b) If $L$ is a single circle that bounds a disk then $\hat{q}_{f}(L)=0$.

(c) If $L_{1}$ and $L_{2}$ are isotopic collections of circles then $\hat{q}_{f}\left(L_{1}\right)=\hat{q}_{f}\left(L_{2}\right)$.

7.4. Pin structure. Throughout this section, let $f: V^{v} \rightarrow \mathrm{R}^{2 v-2}$ be as in Definition 7.16, let $F \subset M_{f}^{\prime}$ be a component of $M_{f}$ and let $v \equiv 3 \bmod 4$.

Proposition 7.18. The immersion $f$ induces a pin structure on $F$ and hence a $\mathrm{Z}_{4}$-quadratic function $q_{f, F}$ on $H_{1}\left(F ; Z_{2}\right)$.

Proof. Note that $\pi_{1}(\mathrm{SO}(2 v-2) / \mathbf{A}(2 v-2)) \cong Z_{4}$ and that $\hat{q}_{f}(c)$ is independent of orientation in this case. Hence $\hat{q}_{f}$ is a function from disjoint unions of circles in $F$ to $\mathrm{Z}_{4}$. Since $\hat{q}_{f}$ satisfies (a) and (b) of Proposition 7.17, we know by Lemma 3.4 in [8] that $\hat{q}_{f}$ induces a $Z_{4}$-quadratic function $q_{f, F}: H_{1}\left(F ; Z_{2}\right) \rightarrow Z_{4}$. By Theorem 3.2 in the same paper there is a $1-1$ correspondence between $Z_{4}$-quadratic functions of the intersection form of $F$ and pin structures.

Let $L^{g}$ be a vector space over $Z_{2}$ with a nonsingular bilinear form $(x, y) \mapsto x \cdot y \in \mathbf{Z}_{2}$. A $\mathbf{Z}_{4}$-quadratic function is a function $q: L \rightarrow \mathbf{Z}_{4}$ such that $q(x+y)=q(x)+q(y)+(x \cdot y) \cdot 2$. Let $q$ be such a function and define the complex number

$$
\lambda(q)=\sum_{x \in L}(\sqrt{-1})^{q(x)},
$$

then $\lambda(q)=\sqrt{2}^{g}\left(\frac{1+\sqrt{-1}}{\sqrt{2}}\right)^{m}$, for some $m \in \mathbf{Z}$. Since $\frac{1+\sqrt{-1}}{\sqrt{2}}$ is an $8^{\text {th }}$ root of unity, the residue class of $m$ modulo 8 is a well defined element in $Z_{8}$. It is denoted $\beta(q)$ and called Brown's invariant of $q$. For any quadratic function $q: L^{g} \rightarrow \mathbf{Z}_{4}, \beta(q) \equiv g \bmod 2$. If $q_{1}$ and $q_{2}$ are two $\mathbf{Z}_{4}$-quadratic functions on $L$ then there is an isomorphism $h$ of $L$, preserving the bilinear form, such that $q_{2}=q_{1} h$ if and only if $\beta\left(q_{1}\right)=\beta\left(q_{2}\right)$. For a reference on this subject see [10].

Definition 7.19. If $v \equiv 3 \bmod 4$ and $F \subset M_{f}^{\prime}$, define $s(F)=\beta\left(q_{f, F}\right) \in Z_{8}$. Let $h: V^{v} \rightarrow \mathrm{R}^{2 v-2}$ be another immersion satisfying the hypothesis in Definition 7.16 . 
Lemma 7.20. Assume that $f$ is diffeotopy equivalent to $h$. Let $F$ be a component of $M_{f}$ and $G$ be the component of $M_{h}$ corresponding to $F$ under the diffeotopy equivalence then $g(F)=g(G)$. If $F \subset M_{f}^{\prime}$ then $G \subset M_{h}^{\prime}$ and $s(F)=s(G)$.

Proof. This follows from Lemma 7.7 and the fact that $K_{1 *} \mid F: H_{1}\left(F ; Z_{2}\right) \rightarrow H_{1}\left(K_{1} F ; Z_{2}\right)$ is an isomorphism.

7.5. $\mathbf{Z}_{2} \oplus \mathbf{Z}_{2}$-quadratic functions. Throughout this section, let $f: V^{v} \rightarrow$ $\mathrm{R}^{2 v-2}$ be as in Definition 7.16, let $F \subset M_{f}^{\prime}$ be a component of $M_{f}$ and let $v \equiv 2 \bmod 4$. To describe the invariants in this case it is necessary to introduce some algebra.

Definition 7.21. Let $\left(L^{2 g}, \cdot\right)$ be a symplectic vector space over $Z_{2}$. A function $q: L \rightarrow \mathbf{Z}_{2} \oplus \mathbf{Z}_{2}$ is called a $\mathbf{Z}_{2} \oplus \mathbf{Z}_{2}$-quadratic function if

$$
q(x+y)=q(x)+q(y)+(x \cdot y) \cdot(1,1), \quad \text { for all } x, y \in L,
$$

where $u \cdot(1,1)$ is the the value at $u \in Z_{2}$ of the homomorphism $Z_{2} \rightarrow Z_{2} \oplus \mathbf{Z}_{2}$ taking 1 to $(1,1)$.

REMARK 7.22. Let $q$ be a $\mathbf{Z}_{2} \oplus \mathbf{Z}_{2}$-quadratic function on $L$. If $p_{i}: \mathbf{Z}_{2} \oplus$ $\mathbf{Z}_{2} \rightarrow \mathbf{Z}_{2}, i=1,2$ is the projection to the $i^{t h}$ summand then $p_{i} q, i=1,2$ are $\mathbf{Z}_{2}$ quadratic functions on $L$.

Definition 7.23. Let $q$ be a $Z_{2} \oplus Z_{2}$-quadratic function on $L^{2 g}$ and $\left\{a_{i}, b_{i}\right\}_{i=1}^{g}$ be a symplectic basis. Define $\operatorname{Arf}(q) \in \mathbf{Z}_{2} \oplus \mathbf{Z}_{2}$ by

$$
\operatorname{Arf}(q)=\sum_{i} q\left(a_{i}\right) q\left(b_{i}\right)
$$

where $(x, y)\left(x^{\prime}, y^{\prime}\right)=\left(x x^{\prime}, y y^{\prime}\right)$ for $(x, y),\left(x^{\prime}, y^{\prime}\right) \in \mathbf{Z}_{2} \oplus \mathbf{Z}_{2}$.

By Remark 7.22 and the properties of the usual Arf invariant, we see that $\operatorname{Arf}(q)$ is invariant under symplectomorphisms of $L$.

Proposition 7.24. The immersion $f$ induces $a Z_{2} \oplus \mathbf{Z}_{2}$-quadratic function $q_{f, F}$ on $H_{1}\left(F ; Z_{2}\right)$.

Proof. The construction of $q_{f, F}$ is based on $\hat{q}_{f}$. We have $\pi_{1}(\operatorname{SO}(2 v-2) /$ $\mathbf{A}(2 v-2)) \cong \mathbf{Z}_{2} \oplus \mathbf{Z}_{2}, F$ orientable and $\hat{q}_{f}(c)$ independent of the orientation of $c$. We view $\hat{q}_{f}$ as a function on disjoint unions of embedded circles. The first step is to show that given a collection $L$ of embedded circles we can find a single circle $K$, homologous to $L$, such that $\hat{q}_{f}(L)=\hat{q}_{f}(K)$. If $L$ has more than one component it is possible to draw an arc connecting two of its components. A regular neighborhood of this arc is a disk $D$ with boundary $L_{1}$. By Proposition 7.17 (b) we have $\hat{q}_{f}\left(L_{1}\right)=0$. Now, $L$ and $L_{1}$ have two pairs of intersection points and if we orient the two components of $L$ so that 
they transverse $D$ in opposite directions and smooth the intersection points we get a new collection $K_{1}$ with one component less than $L$ and two boundaries of disks. Now, $K_{1}$ is clearly homologous to $L$ and (a) and (b) of Proposition 7.17 shows that $\hat{q}_{f}(L)=\hat{q}_{f}\left(K_{1}\right)$. Continue this process until there is only one component left.

Assume that $L_{1}$ and $L_{2}$ are homologous. We must show that $\hat{q}_{f}\left(L_{1}\right)=$ $\hat{q}_{f}\left(L_{2}\right)$. By the above we can assume that $L_{1}$ and $L_{2}$ are embedded circles and by Proposition 7.17 (c) and the fact that $F$ is orientable, that they intersect transversely in an even number of points. Smoothing the intersections and applying the above construction we get one zero homologous circle $L$ and $\hat{q}_{f}(L)=\hat{q}_{f}\left(L_{1}\right)+\hat{q}_{f}\left(L_{2}\right)$ so we are done if we can show that $\hat{q}_{f}(L)=0$. Since $L$ is zero homologous there is a surface $W$, with $\partial W$, a single circle and an embedding $W \rightarrow F$ taking $\partial W$ to $L$. If $W$ is a disk then $\hat{q}_{f}(L)=0$. We proceed by induction on the Euler characteristic of $W$. If $\chi(W)<1$ there is a handle in $W$. Let $K_{1}$ and $K_{2}$ be two disjoint meridians of this handle then $\hat{q}_{f}\left(K_{1}\right)=\hat{q}_{f}\left(K_{2}\right)$. Connect both of these to $L$ by disjoint arcs and apply the first construction of the proof twice. We then get a curve $L^{\prime}$ that bounds a surface $W^{\prime}$ with $\chi\left(W^{\prime}\right)>\chi(W)$ and $\hat{q}_{f}\left(L^{\prime}\right)=0$ by our inductive assumption. Finally,

$$
\hat{q}_{f}\left(L^{\prime}\right)=\hat{q}_{f}\left(K_{1}\right)+\hat{q}_{f}\left(K_{2}\right)+\hat{q}_{f}(L),
$$

hence $\hat{q}_{f}(L)=0$. Define $q_{f, F}: H_{1}\left(F ; \mathbf{Z}_{2}\right) \rightarrow \mathbf{Z}_{2} \oplus \mathbf{Z}_{2}$ by $q_{f, F}(a)=\hat{q}_{f}(\alpha)$ where $\alpha$ is an embedded circle with $[\alpha]=a$. Then $q_{f, F}$ is a $\mathbf{Z}_{2} \oplus \mathbf{Z}_{2}$-quadratic function.

Definition 7.25. If $v \equiv 2 \bmod 4$ and $F \subset M_{f}^{\prime}$, define $s(F)=\operatorname{Arf}\left(q_{f, F}\right) \in$ $\mathrm{Z}_{2} \oplus \mathrm{Z}_{2}$.

Let $h: V^{v} \rightarrow \mathrm{R}^{2 v-2}$ be another immersion satisfying the hypothesis in Definition 7.16.

Lemma 7.26. Assume that $f$ is diffeotopy equivalent to $h$. Let $F$ be a component of $M_{f}$ and $G$ be the component of $M_{h}$ corresponding to $F$ under the diffeotopy equivalence then $g(F)=g(G)$. If $F \subset M_{f}^{\prime}$ then $G \subset M_{h}^{\prime}$ and $s(F)=s(G)$.

Proof. See Lemma 7.20.

Let $L^{2 g}$ be a symplectic vector space over $Z_{2}$. Let $q_{1}, q_{2}$ be $Z_{2} \oplus \mathbf{Z}_{2}$-quadratic functions on $L$ such that there are subspaces $L_{i}^{2 g-1} \subset L$ with $q_{i}\left(L_{i}\right) \subset\{(0,0),(1,1)\}$ and elements $a^{i} \in V$ such that $q_{i}\left(a^{i}\right) \in\{(1,0),(0,1)\}$, $i=1,2$. 
Lemma 7.27. Under the conditions described above there is a symplectomorphism $h: L \rightarrow L$ with $q_{2}=q_{1} h$ if and only if $\operatorname{Arf}\left(q_{1}\right)=\operatorname{Arf}\left(q_{2}\right)$.

Proof. Checking all possible cases proves the lemma if $g \leq 2$. Assume that $g>2$. Pick bases $\left\{a_{1}^{i}, b_{1}^{i}, \ldots, a_{g}^{i}, b_{g}^{i}\right\}$ such that $q_{i}\left(a_{1}^{i}\right)=(1,0)$ or $q_{i}\left(a_{1}^{i}\right)=(0,1)$. Without loss of generality we can assume that $q_{i}\left(a_{j}^{i}\right)=q_{i}\left(b_{j}^{i}\right)=(0,0)$ for $3 \leq j \leq g, i=1,2$. The situation then reduces to the case $g=2$.

ReMark 7.28. When $q_{f, F}: H_{1}\left(F ; Z_{2}\right) \rightarrow Z_{2} \oplus Z_{2}$ is a quadratic function induced by a generic immersion, we have exactly the situation described above Lemma 7.27, since $\hat{q}_{f}(c) \notin\{(0,0),(1,1)\}$ if and only if $\widetilde{c}$ is connected and $\widetilde{c}$ is connected if and only if $[c] \notin f_{*}\left(H_{1}\left(\widetilde{F} ; \mathbf{Z}_{2}\right)\right)$.

7.6. Absence of local obstructions. Throughout this section let $f: V^{v} \rightarrow$ $\mathrm{R}^{2 v-2}$ be as in Definition 7.16, let $F \subset M_{f}^{\prime}$ be a component of $M_{f}$ and let $v \equiv 0 \bmod 4$ or $v \equiv 1 \bmod 4$. Theorem 7.30 shows that if $v \equiv 0 \bmod 4$ then $g(F)$ is even.

LeMma 7.29.

(a) If $v \equiv 1 \bmod 4$ then there is a disjoint collection of oriented embedded curves in $F,\left\{\alpha_{1}, \ldots, \alpha_{g}\right\}$, such that $\left\{\left[\alpha_{1}\right], \ldots,\left[\alpha_{g}\right]\right\}$ is a basis of $H_{1}\left(F ; Z_{2}\right)$ and $\hat{q}_{f}\left(\alpha_{i}\right)=(1,0)$ for all $i$.

(b) If $v \equiv 0 \bmod 4$ then there is a collection of oriented embedded curves in $F,\left\{\alpha_{1}, \beta_{1}, \ldots, \alpha_{g}, \beta_{g}\right\}$, such that $\alpha_{i} \cap \beta_{i}$ is exactly one point for each $i$ and these are the only intersections in the collection. Also, $\left\{\left[\alpha_{1}\right],\left[\beta_{1}\right], \ldots,\left[\alpha_{g}\right],\left[\beta_{g}\right]\right\}$ is a basis of $H_{1}(F ; Z),\left[\alpha_{i}\right] \cdot\left[\beta_{i}\right]=1$ and $\hat{q}_{f}\left(\alpha_{i}\right)=\hat{q}_{f}\left(\beta_{i}\right)=1$, for all $i$.

Proof. Consider case (a). Let $\alpha_{i}$ be the core of the $i^{\text {th }}$ Moebius strip in $F$ then the basis statement follows. Since $\widetilde{F} \rightarrow F$ is the orientation double cover

$$
\hat{q}_{f}\left(\alpha_{i}\right)=(1,0), \quad \text { or } \quad \hat{q}_{f}\left(\alpha_{i}\right)=(0,1) .
$$

If $-\alpha_{i}$ denotes $\alpha_{i}$ with the opposite orientation then

$$
\hat{q}_{f}\left(-\alpha_{i}\right)=\hat{q}_{f}\left(\alpha_{i}\right)+(1,1),
$$

by Lemma 7.9. Then (a) follows.

Consider case (b). We see that $F \approx \Sigma_{g}$, some $g$. We can pick embedded curves $\left\{\gamma_{1}, \delta_{1}, \ldots, \gamma_{g}, \delta_{g}\right\}$ as in the statement of the theorem, such that $\widetilde{\gamma}_{1}$ is connected, and the inverse image of any other curve in the collection is disconnected. Now, let $D_{i}$ denote a Dehn twist along $\delta_{i}, i>1$. Let $D$ denote a Dehn twist along a curve in the class $\sum_{i=1}^{g}\left[\gamma_{i}\right]$ and $h=D D_{2} \ldots D_{g}$. Then

$$
\hat{q}_{f}\left(h \gamma_{i}\right)= \pm 1 \quad \text { and } \quad \hat{q}_{f}\left(h \delta_{i}\right)= \pm 1 .
$$


If, for example, $\hat{q}_{f}\left(h \gamma_{i}\right)=1$ and $\hat{q}_{f}\left(h \delta_{i}\right)=-1$ then let $\alpha_{i}=-h \delta_{i}$ and $\beta_{i}=h \gamma_{i}$. The other cases that arise can be treated similarly. We get the desired collection $\left\{\alpha_{1}, \beta_{1}, \ldots, \alpha_{g}, \beta_{g}\right\}$. (Note that there is a diffeomorphism $k: F \rightarrow F$ such that $k \gamma_{i}=\alpha_{i}$ and $k \delta_{i}=\beta_{i}$, for all $i$.)

As we shall see in Section 7.7, Lemma 7.29 implies that there are no local obstructions to diffeotopy equivalence associated to components $F \subset M_{f}^{\prime}$ if $v \equiv 0 \bmod 4$. We therefore define $s(F)=0$ in this case. If $v \equiv 1 \bmod 4$ and $F \subset M_{f}^{\prime}$ then there is a local obstruction in $\mathrm{Z}_{2}$ : Proposition 2.8 shows that there is an induced spin structure on $\widetilde{F}=f^{-1}(F)$. Let $q_{f, \widetilde{F}}$ denote the corresponding quadratic function. Since $F$ is nonorientable, there is a unique torsion class $a \in H_{1}(F ; \mathbf{Z})$. Since $g(F)$ is even, there is an element $b^{\prime} \in H_{1}(\widetilde{F} ; \mathbf{Z})$ such that $f_{*}\left(b^{\prime}\right)=a$. Moreover, reducing $b^{\prime}$ modulo 2 gives a uniquely determined $b \in H_{1}\left(\widetilde{F} ; \mathbf{Z}_{2}\right)$. Define

$$
s(F)=q_{f, F}^{\sim}(b) .
$$

Then $s(F)$ is invariant under diffeotopy equivalence. Noting that $f_{*}(b)$ is dual to $w_{1}$, we see that $s(F)=s\left(F^{\prime}\right)$ is a sufficient condition for the existence of the desired diffeomorphism $p: F^{\prime} \rightarrow F$ in the proof below.

7.7. Proof of Theorem 1.5. The only if part follows from Lemmas 7.3, 7.20, 7.26. When $F \subset M_{f}^{\prime \prime}$, we let $\hat{q}_{f}(\alpha)=q_{f, F}([\alpha])$ for embedded curves $\alpha$ in $F$. Here $q_{f, F}$ is the $Z_{2}$-quadratic form associated to the spin structure induced on $F$ and $[\alpha]$ is the $\mathrm{Z}_{2}$-homology class of $\alpha$. With this convention we can treat all cases simultaneously.

Assume that $F^{\prime}$ is a component of $M_{f}$ and that $F$ is a component of $M_{h}$ such that $\widetilde{F}$ and $\widetilde{F}^{\prime}$ have the same number of components, $g(F)=g\left(F^{\prime}\right)$ and $s\left(F^{\prime}\right)=s(F)$. If $G$ is a surface then any automorphism of $H_{1}\left(G ; Z_{2}\right)$ that preserves the intersection pairing lifts to a autodiffeomorphism of $G$. Using this and Lemmas 7.27, 7.29 we see that there is a diffeomorphism $p: F^{\prime} \rightarrow F$ such that $\hat{q}_{f}\left(p^{-1}\left(\alpha_{i}\right)\right)=\hat{q}_{h}\left(\alpha_{i}\right)$, where $\left\{\left[\alpha_{i}\right]\right\}$ is a basis of $\mathbf{Z}_{2}$-homology. By transversality arguments any two embeddings of $F$ in $\mathrm{R}^{2 v-2}$ are ambient diffeotopic. Hence, there is a diffeotopy $K_{t}: \mathrm{R}^{2 v-2} \rightarrow \mathrm{R}^{2 v-2}$ such that $K_{1} \mid F^{\prime}=p$. Since $\hat{q}(\alpha)$ determines how $\alpha$ lifts and $\hat{q}_{f} p^{-1}$ agrees with $\hat{q}_{h}$ on a basis of $\mathrm{Z}_{2}$-homology, the diffeomorphism $p^{-1}: F \rightarrow F^{\prime}$ is covered by a diffeomorphism $k: \widetilde{F} \rightarrow \widetilde{F}^{\prime}$. That is, we have the commutative diagram

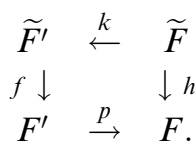

A similar argument as above shows that we can find a diffeotopy $G_{t}: V \rightarrow V$ such that $G_{1} \mid \widetilde{F}=k$. It follows that $K_{1} f G_{1}|\widetilde{F}=h| \widetilde{F}$. Let $K_{1} f G_{1}=f^{\prime}$. We can 
find diffeotopies $P_{t}$ and $P_{t}^{\prime}$, fixed on $F$, of $\mathrm{R}^{2 v-2}$ such that the normal derivatives of $P_{1} h$ and $P_{1}^{\prime} f^{\prime}$ are constant along the fibers of tubular neighborhoods of $h^{-1}\left(\alpha_{i}\right)$ in $\widetilde{F}$. We therefore assume that $f^{\prime}$ and $h$ have this property. Lemma 2.14 shows that we can replace $f^{\prime}$ and $h$ by their normal derivatives close to $\widetilde{F}$. Choose framings of $N(\widetilde{F} \subset V)$ along the curves $f^{-1}\left(\alpha_{i}\right)=\widetilde{\alpha}_{i}$. Then $f^{\prime}$ and $h$ induce framings or twist framings of $N\left(F \subset \mathrm{R}^{2 v-2}\right) \mid \alpha_{i}$. By Lemmas 7.7, 7.2, $\hat{q}_{f^{\prime}}\left(\alpha_{i}\right)=\hat{q}_{h}\left(\alpha_{i}\right)$. This means that the induced framings or twist framings are isotopic. Clearly, we can assume that $F$ is fixed under such an isotopy. We now separate the cases.

First, assume that $F$ is orientable, $g(F)>0$ and that the basic curves are $\left\{a_{1}, b_{1}, \ldots, a_{g}, b_{g}\right\}$. Choose a diffeotopy $L_{t}^{1}$ of $\mathrm{R}^{2 v-2}$, supported in a neighborhood of $a_{1}$, that brings the framing of $a_{1}$ inducedby $f^{\prime}$ to that induced by $h$ and that keeps $F$ fixed. Choose a second diffeotopy $J_{t}^{1}$, supported in a neighborhood of $b_{1}$ and fixed on $F$, keeping the framing at the intersection point of $a_{1}$ and $b_{1}$ fixed, and such that it brings the framing induced by $f^{\prime}$ on $b_{1}$ to that induced by $h$. Let $c_{1}$ be a path connecting $a_{1}$ to $a_{2}$. Choose a diffeotopy $R_{t}^{1}$ supported in a neighborhood of $c_{1}$ with the same features as the diffeotopies already used. Continue in this manner until $b_{g}$ is reached. Then $R_{1}=R_{1}^{g} L_{1}^{g} J_{1}^{g-1} \ldots J_{1}^{1} L_{1}^{1} R_{1}^{1}$ is one end of a diffeotopy of $\mathrm{R}^{2 v-2}$ such that $R_{1} f^{\prime}|\widetilde{F}=h| \widetilde{F}$ and $R_{1} f^{\prime}$ and $h$ are equal in $N\left(F \subset \mathrm{R}^{2 v-2}\right) \mid \widetilde{C}$, where $\widetilde{C}$ is a neighborhood in $\widetilde{F}$ of $h^{-1}\left(\widetilde{a}_{1} \cup \widetilde{b}_{1} \cup \widetilde{c}_{1} \cup \ldots \cup \widetilde{b}_{g}\right)$. Now, $F-C$, where $C=h(\widetilde{C})=R_{1} f^{\prime}(\widetilde{C})$ is a disk $D$ with boundary $\partial D=\partial C$. The maps $R_{1} f^{\prime}$ and $h$ induce framings of this disk, from a chosen framing of $N(\widetilde{D} \subset V)$. These framings agree on $\partial D$. If we can find a homotopy between the two framings then a diffeotopy $L_{t}$ of $\mathrm{R}^{2 v-2}$ such that $L_{1} R_{1} f^{\prime}=h$ in a neighborhood of $\widetilde{F}$ is easily found. Interpreting the framings in the usual way we must look for a solution of the homotopy problem

$$
\begin{array}{ccc}
D \times \partial I \cup \partial D \times I & \rightarrow & \mathrm{SO}(2 v-4) \\
D \times I & &
\end{array}
$$

Since $\pi_{2}(\operatorname{SO}(2 v-4))=0$, there is a solution.

Secondly, if $F$ is nonorientable we construct the desired diffeotopy in almost the same way, just drop the $b$ 's and $J$ 's in the above construction. Finally, if $g(F)=0$ we start from any simple closed curve $a$ in $F$ and get two pairs of framed disks with coinciding boundary framings. We see, as above, that the desired diffeotopy can be constructed.

Repeating this construction for any component of $M_{h}$ we can find diffeotopies $K_{t}: \mathrm{R}^{2 v-2} \rightarrow \mathrm{R}^{2 v-2}$ and $G_{t}: V \rightarrow V$ such that $K_{1} f G_{1}|W=h| W$ where $W$ is a neighborhood of $\widetilde{M}_{h}$. An application of Theorem 1.2 completes the proof. 


\subsection{Restrictions on the self intersection.}

Theorem 7.30. Let $f: V^{v} \rightarrow \mathrm{R}^{2 v-2}$ be as in Definition 7.16 and let $v \equiv 1 \bmod 4$. If $F \subset M_{f}^{\prime}$ is a component of $M_{f}$ then $g(F)$ is even.

Proof. Note that $F \approx \Gamma_{g}$. Assume, to get a contradiction, that $g$ is odd. Pick an embedded curve $\alpha \subset F$ that is dual to the first Stiefel-Whitney class $w_{1}$. Since $g$ is odd, a regular neighborhood of $\alpha$ is a Moebius strip, $B$. Since $\alpha$ was dual to $w_{1}, F-B$ is an orientable surface with a single boundary circle $c=\partial B$. The covering $f: \widetilde{F} \rightarrow F$ is the orientation double covering, therefore $f^{-1}(F-B)$ is disconnected and hence there is an induced spin structure on $F-B$. This implies that the spin structure induced by $f$ on $T F \mid c$ must be the trivial one, which in turn implies that

$$
\left\langle\xi_{(F-B)},\left[\vec{c}_{F-B}\right]\right\rangle=1,
$$

by Remark 2.13. Noting that $\widetilde{c}$ is disconnected it is easy to see (cf. Lemma 7.10) that

$$
\omega_{f}(c)=0 \text { if and only if }\left\langle\xi_{(F-B)},\left[\vec{c}_{F-B}\right]\right\rangle=1 .
$$

However, using Lemma 7.13,

$$
\begin{aligned}
\omega_{f}(c) & =\omega_{f}(\alpha)+\omega_{f}(\alpha)+p_{*}(u) \\
& =p_{*}(u) \neq 0,
\end{aligned}
$$

since $\pi_{1}(\mathbf{S O}(2 v-2) / \mathbf{A}(2 v-2)) \cong \mathbf{Z}_{2} \oplus \mathbf{Z}_{2}$ when $v \equiv 1 \bmod 4$. This is a contradiction.

The next theorem shows that there are similar restrictions on the self intersection in other dimensions.

THEOREM 7.31. Let $M^{3}$ be any closed orientable 3-manifold. If $f: M^{3} \rightarrow S^{4}$ is an immersion with transverse self intersection consisting of double points (i.e. there are no triple points), then there are no nonorientable components $F$ of $M_{f}$ with odd genus.

Proof. Assume that $f: M^{3} \rightarrow S^{4}$ is an immersion with transverse self intersection along $F$ then $f^{-1}(F)=\widetilde{F}$ is an orientable surface by Proposition 2.5. The normal bundle of $\widetilde{F}$ in $M^{3}$ is orientable and therefore trivial. If $n$ is a nonvanishing normal vector field of $\widetilde{F}$ in $M^{3}$ then define the normal vector field $v$ of $F$ in $S^{4}$ by

$$
v(x)=d f\left(n\left(x_{1}\right)\right)+d f\left(n\left(x_{2}\right)\right),
$$

where $f\left(x_{1}\right)=f\left(x_{2}\right)=x$. By the assumption about transversality, this vector field never vanishes. If $g(F)$ is odd then this contradicts the fact, proved in 
[9], that the Euler number of the normal bundle of an embedded nonorientable surface $G$ in $S^{4}$ is congruent to $2 \chi(G)$ modulo 4 .

\section{Examples of generic immersions.}

\subsection{Self intersections along spheres.}

ExAmple 8.1. There is a generic immersion $f: S^{v} \rightarrow \mathrm{R}^{w}$ such that $M_{f} \approx S^{2 v-w}$ and $\widetilde{M}_{f} \approx S^{2 v-w} \cup S^{2 v-w}$.

Let $S^{v} \subset \mathrm{R}^{v+1} \times 0 \subset \mathrm{R}^{w}$ be a sphere of radius 1. Let $D^{v} \subset 0 \times \mathrm{R}^{v} \subset \mathrm{R}^{w}$ be a disk of radius 2. Then $S^{v} \cap D^{v}=S^{2 v-w}$ is a sphere of radius 1 . Cut out a small $v$-disk, disjoint from the intersection, from $S^{v}$. Join the boundary of this disk and $\partial D^{v}$ by a $v$-tube. What is obtained can be viewed as the image of a generic immersion with the required properties.

\subsection{Self intersections along circles.}

ExAmple 8.2. There is an immersion $f: S^{5} \rightarrow \mathrm{R}^{9}$ with transverse self intersection $M_{f} \approx S^{1}$ and $\widetilde{M}_{f} \approx S^{1}$.

To construct the map, consider the following decomposition

$$
S^{5}=S_{a}^{1} \times D_{a}^{4} \cup D_{b}^{2} \times S_{b}^{3} .
$$

Choose a path $\lambda:[0,1] \rightarrow \mathrm{SO}(8)$ that is constant near the ends, starting at the identity matrix $\mathbf{I}$ and ending at the matrix $\mathbf{B}(8)$. We denote the column vectors in the matrix $\lambda(t)$ by $F^{i}(t), i=1, \ldots, 8$. Let $\theta$ be a coordinate on $S_{a}^{1}$ ranging from 0 to 1 and $\left(x_{1}, x_{2}, x_{3}, x_{4}\right) \in \mathbf{D}^{4}$, the unit disk in $\mathbf{R}^{4}$, be coordinates on $D_{a}^{4}$. Choose coordinates on $S^{1} \times D^{8}$ as $(\omega, y)$ where $0 \leq \omega \leq 1$ and $y \in \mathbf{D}^{8}$, the unit disk in $\mathbf{R}^{8}$. Define the map $f^{\prime}: S_{a}^{1} \times D_{a}^{2} \rightarrow S^{1} \times D^{8}$ by

$$
f^{\prime}(\theta, x)= \begin{cases}\left(2 \theta, \sum_{i=1}^{4} x_{i} F^{i}(2 \theta)\right) & \text { if } 0 \leq \theta \leq \frac{1}{2}, \\ \left(2 \theta-1, \sum_{i=1}^{4} x_{i} F^{i+4}(2 \theta-1)\right) & \text { if } \frac{1}{2} \leq \theta \leq 1 .\end{cases}
$$

Then $f^{\prime}$ has transverse self intersection along $S^{1} \times 0$ and $f^{\prime-1}\left(S_{c}^{1} \times 0\right)=$ $S_{a}^{1} \times 0$. To complete the construction, we consider $S^{1} \times D^{8}$ as a submanifold of $\mathrm{R}^{9}$ and extend $f^{\prime}$ to $D_{b}^{2} \times S_{b}^{3}$ as an embedding of this space into $\mathrm{R}^{9}-S^{1} \times D^{8}$. Note that $f^{\prime}$ is already defined on $\partial D_{b}^{2} \times S_{b}^{3}$ and embedds this boundary into $\partial\left(\mathrm{R}^{9}-S^{1} \times D^{8}\right)$. Standard obstruction theory shows that this extends to a map $g$ from $D_{b}^{2} \times S_{b}^{3}$ to $\mathrm{R}^{9}-S^{1} \times D^{8}$. Then Theorem 3.1 shows that $g$ is actually homotopic relative to the boundary to an embedding $f^{\prime \prime}$. By the analogue of Theorem 3.5 for embeddings, we can assume that $f^{\prime \prime}$ is smooth and then use it to extend $f^{\prime}$. This gives the desired $f$. 
8.3. Self intersections along orientable surfaces. Let $T^{2}$ denote the standard torus.

EXAMPLE 8.3. There is a generic immersion $f: S^{4} \rightarrow \mathrm{R}^{6}$ with $M_{f} \approx T^{2}$ and $\widetilde{M}_{f} \approx T^{2} \cup T^{2}$.

Consider $\mathbf{D}^{4}$, the unit disk in $\mathbf{C}^{2}$, with coordinates $(z, w)$ and the embeddings $f_{i}: \mathbf{D}^{4} \rightarrow \mathrm{C}^{2} \times \mathrm{R}^{2}, i=1,2$, defined by

$$
\begin{aligned}
& f_{1}(z, w)=(z, w, 0,0), \\
& f_{2}(z, w)= \\
& \left(z, w, \frac{1}{4}-|z|^{2}-\alpha\left(|z|^{2}+|w|^{2}\right)|w|^{2}, \frac{1}{4}-|w|^{2}-\alpha\left(|z|^{2}+|w|^{2}\right)|z|^{2}\right),
\end{aligned}
$$

where $\alpha:[0,1] \rightarrow[0,1]$ satisfies $0 \leq \alpha \leq 1, \alpha(t)=0$ if $0 \leq t \leq \frac{3}{4}$ and $\alpha(t)=1$ if $\frac{7}{8} \leq t \leq 1$. We see that $f_{1}\left(\mathbf{D}^{4}\right) \cap f_{2}\left(\mathbf{D}^{4}\right) \approx T^{2}$ and that the self intersection is transverse. Now, for $x \in \partial \mathbf{D}^{4}$ we have

$$
f_{2}(x)=f_{1}(x)-\left(0,0,-\frac{1}{2},-\frac{1}{2}\right) .
$$

Hence, we can connect the two disks by an embedding of $S^{3} \times I$. This gives us the desired $f$.

EXAmple 8.4. There is a generic immersion $f: S^{6} \rightarrow \mathrm{R}^{10}$ with $M_{f} \approx T^{2}$ and $\widetilde{M}_{f} \approx T^{2}$.

The construction of this map involves the path $\lambda$ described in Example 8.2. We keep the notation. Consider closed tubular neighborhoods of embedded tori in $S^{6}$ and $\mathrm{R}^{10}$, denote them by $U$ and $V$ respectively. They are of the form $U=S^{1} \times S^{1} \times D^{4}$ and $V=S^{1} \times S^{1} \times D^{8}$. Let $(\theta, \omega)$ be coordinates on $S^{1} \times S^{1},\left(x_{1}, \ldots, x_{4}\right) \in \mathbf{D}^{4}$ coordinates on $D^{4}$ and $v \in \mathbf{D}^{8}$ be coordinates on $D^{8}$. Define the map $\phi: U \rightarrow V$ by

$$
f^{\prime}(\theta, \omega, x)= \begin{cases}\left(2 \theta, \omega, \sum_{i=1}^{4} x_{i} F_{i}(2 \theta)\right) & \text { for } 0 \leq \theta \leq \frac{1}{2} \\ \left(2 \theta-1, \omega, \sum_{i=1}^{4} x_{i} F_{i+4}(2 \theta-1)\right) & \text { for } \frac{1}{2} \leq \theta \leq 1\end{cases}
$$

It is straightforward to check that this map has transverse self intersection $M_{f}=S^{1} \times S^{1} \times 0 \subset V$ and that $f^{-1}\left(M_{f}\right)=S^{1} \times S^{1} \times 0 \subset U$. To complete the construction we shall extend the map to $S^{6}-U$ as an embedding into $\mathrm{R}^{10}-V$. First we apply obstruction theory to see that it extends as a continuous map. The key fact is that $S^{10}-T^{2}$ is 6-connected, so that all obstructions vanish. When this is done we have a continuous map $f^{\prime \prime}: S^{6}-U \rightarrow S^{10}-V$ that is a smooth embedding close to the boundary. Then we apply Theorem 3.1 to see that it is homotopic to an embedding. The key facts here are that $S^{6}-T^{2}$ is 2-connected and $\operatorname{dim}\left(S^{10}-V\right)-$ 
$\operatorname{dim}\left(S^{6}-U\right)=4>3$. Then proceed as in Example 8.2 to get the desired map.

8.4. Self intersections along nonorientable surfaces. Theorem 7.30 shows that a nonorientable surface, which is a self intersection of a generic immersion, must have even genus if the dimension $v$ of the source manifold satisfies $v \equiv 1 \bmod 4$. The following example shows that the condition of genericity is necessary.

Example 8.5. Denote the coordinates in $\mathbf{R}^{8}$ by $y=\left(y_{1}, \ldots, y_{8}\right)$ and consider $S^{5}$ as the unit sphere in $\mathrm{R}^{6}$ with coordinates $\left(x_{1}, \ldots, x_{6}\right)$. Define the family of maps $f_{\epsilon}: S^{5} \rightarrow \mathbf{R}^{8},-1<\epsilon<1$, by the equations

$$
\begin{array}{ll}
y_{1}=x_{1}^{2}-x_{2}^{2}, & y_{5}=x_{3}\left(x_{4}^{2}+x_{5}^{2}+x_{6}^{2}+\epsilon^{2}\right), \\
y_{2}=x_{1} x_{2}, & y_{6}=x_{4}, \\
y_{3}=x_{1} x_{3}-x_{2}\left(x_{4}^{2}+x_{5}^{2}+x_{6}^{2}+\epsilon^{2}\right), & y_{7}=x_{5}, \\
y_{4}=x_{2} x_{3}+x_{1}\left(x_{4}^{2}+x_{5}^{2}+x_{6}^{2}+\epsilon^{2}\right), & y_{8}=x_{6} .
\end{array}
$$

If $\epsilon \neq 0$ then $f_{\epsilon}$ is an embedding: The last three equations determine $x_{4}, x_{5}, x_{6}$, the fifth then determines $x_{3}$. Finally, considering $x_{3}, x_{4}, x_{5}, x_{6}$ as fixed, the third and fourth equation form a linear system in $x_{1}, x_{2}$ with nonzero determinant. Similarly, $f_{0}$ is injective on the subset $\left(x_{4}^{2}+x_{5}^{2}+x_{6}^{2} \neq 0\right)$ of $S^{5}$. The restriction $f_{0} \mid\left(x_{4}=x_{5}=x_{6}=0\right): S^{2} \subset S^{5} \rightarrow \mathrm{R}^{4} \subset \mathrm{R}^{8}$ is an immersion with image $\mathrm{R} P^{2}$. Thus, $f_{0}: S^{5} \rightarrow \mathrm{R}^{8}$ is an immersion with self intersection $\mathrm{R} P^{2}$. However, an arbitrarily small perturbation of $f_{0}$ gives a map $f_{\epsilon}$, which is an embedding. Hence, the self intersection is not transverse.

Example 8.6. There is a generic immersion $f: S^{7} \rightarrow \mathrm{R}^{12}$ with $M_{f} \approx \mathrm{R} P^{2}$ and $\widetilde{M}_{f} \approx S^{2}$.

To construct this map we write

$$
S^{7}=S_{a}^{1} \times D_{a}^{6} \cup D_{b}^{2} \times S_{b}^{5}
$$

Then we choose a path $\lambda:[0,1] \rightarrow \mathrm{SO}(11)$, starting at the identity $\mathbf{I}$ and ending at the matrix $\mathbf{B}(11)$. We denote the column vectors of the matrix $\lambda(t)$ by $F^{i}(t), i=1, \ldots, 11$. Let $(\theta, x) \in[0,1] \times \mathbf{D}^{6}$ be coordinates on $S_{a}^{1} \times D_{a}^{6}$. Let $(\omega, v) \in[0,1] \times \mathbf{D}^{11}$ be coordinates on $S^{1} \times D^{11} \subset \mathrm{R}^{12}$. Let $K$ denote the manifold $\mathrm{R}^{12}-S^{1} \times D^{11}$. Define the map $f^{\prime}: S_{a}^{1} \times D_{a}^{6} \rightarrow S^{1} \times D^{11}$ by

$$
f^{\prime}(\theta, x)=\left\{\begin{array}{l}
\left(2 \theta, x_{1} F_{1}(2 \theta)+\sum_{i=2}^{6} x_{i} F_{i}(2 \theta)\right), \\
\left(2 \theta-1,-x_{1} F_{1}(2 \theta-1)+\sum_{i=2}^{6} x_{i} F_{i+5}(2 \theta-1)\right),
\end{array}\right.
$$

where the first equation holds for $0 \leq \theta \leq \frac{1}{2}$ and the secondfor $\frac{1}{2} \leq \theta \leq 1$. The self intersection manifold of $f^{\prime}$ is a Moebius strip $B$ with boundary circle $\partial B$ 
in $S^{1} \times \partial D^{11}$. The inverse image of this set is the band $S_{a}^{1} \times I \times 0$ where $I$ is the unit interval in the $x_{1}$-direction in $\mathbf{D}^{6}$. Moreover, the intersection is transverse. The next step is to extend $f^{\prime}$ to $D_{b}^{2} \times S_{b}^{5}$. To this end we write

$$
D_{b}^{2} \times S_{b}^{5}=D_{b}^{2} \times\left(D_{n}^{5} \cup S^{4} \times I \cup D_{s}^{5}\right) .
$$

Here $S_{b}^{5}$ is considered as the boundary of $D_{a}^{6}$ and $D_{n}^{5}$ is a small disk centered on the point $(1,0,0,0,0,0)$ in the coordinates above, $D_{s}^{5}$ is the antipodal disk. The circle $\partial B \subset \partial K$ is homotopically trivial in $K$ and hence bounds a 2disk. By transversality arguments there is actually an embedded disk $E^{2} \subset K$ with $\partial E^{2}=\partial B$. Moreover, $E^{2}$ can be chosen so that $d f^{\prime}(\theta, \pm 1,0,0,0,0,0) \frac{\partial}{\partial x_{1}}$ is an outward normal vector field to $\partial E^{2}$. The framing induced on $\partial E$ by $f^{\prime}$ induces a loop of the form $\lambda * \lambda \mathbf{A}(12)$ in $\mathrm{SO}(12)$, where $\lambda:[0,1] \rightarrow \mathrm{SO}(12)$ is a path from $\mathbf{I}$ to $\mathbf{A}(12)$. Since $\pi_{1}(\mathbf{S O}(12) / \mathbf{A}(12)) \cong \mathbf{Z}_{4}$ this is the nontrivial loop. This means that the normal framing of $\partial E$ extends to a normal framing of $E$. At this point we can find an extension $f^{\prime \prime}$ of $f^{\prime}$ to $D_{b}^{2} \times\left(D_{n}^{5} \cup D_{s}^{5}\right)$. Namely, map $D_{b}^{2} \times n$ and $D_{b}^{2} \times s$ onto $E^{2}$. Take the five vectors of $D_{n}^{5}$ to the first five vectors in the extended frame and the five of $D_{s}^{5}$ to the last five. Then $f^{\prime}$ is a map with transverse self intersection manifold $\mathrm{R} P^{2}$ and we need only extend it as an embedding to $D_{b}^{2} \times S^{4} \times I$ into $W=K-N$, where $N$ is a tubular neighborhood of $E$ in $K$. Note that $f^{\prime}$ is already defined on $\partial D_{b}^{2} \times S^{4} \times I \cup D_{b}^{2} \times S^{4} \times \partial I$ so we meet first the extension problem

$$
\begin{array}{cccc}
\partial D_{b}^{2} \times S^{4} \times I \cup D_{b}^{2} \times S^{4} \times \partial I & \underset{\nearrow}{f^{\prime}} & W \\
& \stackrel{\nearrow}{\longrightarrow} & \\
D_{b}^{2} \times S^{4} \times I & &
\end{array}
$$

The space $W$ is a deformation retract of $K-E$ and $K$ is 10 -connected by general position. Hence, again by general position we see that $W$ is 9-connected. Obstructions to extending this map are found in $H^{q+1}\left(S^{4} \times D^{3}, S^{4} \times \partial D^{3} ; \pi_{q}(W)\right)$ and thus vanish. Pick an extension $g$. Then $g$ is an embedding close to the boundary. The argument from Example 8.2 can now be repeated to find the desired $f$.

Example 8.7. There is a generic immersion $f: S^{9} \rightarrow \mathrm{R}^{16}$ such that $M_{f} \approx K^{2}$ and $\widetilde{M}_{f} \approx T^{2}$, where $K^{2}$ denotes the Klein bottle.

Choose a path $\lambda:[0,1] \rightarrow \mathbf{S O}(15)$ such that $\lambda(0)=\mathbf{I}$ and $\lambda(1)=\mathbf{B}(15)$. As in Example 8.6, we can construct a map $g: S^{1} \times D^{8} \rightarrow S^{1} \times D^{15}$ such that $M_{g}=B$, where $B$ is a Moebius strip with $\partial B \subset S^{1} \times \partial D^{15}$. Furthermore, $g^{-1}(B)=\widetilde{B}=S^{1} \times I \times 0 \subset S^{1} \times D^{8}$. Let $\mathrm{R}_{i+}^{n}=\left\{x \in \mathrm{R}^{n}: x_{i} \geq 0\right\}$ and $\mathrm{R}_{i-}^{n}=$ $\left\{x \in \mathrm{R}^{n}: x_{i} \leq 0\right\}$. There is a natural inclusion $\mathrm{R}^{9} \subset S^{9}$. Choose an embedding $\phi: S^{1} \times D^{8} \rightarrow \mathrm{R}_{9+}^{9}$ such that $\phi\left(S^{1} \times 0\right) \subset \mathbf{R}_{9+}^{9} \cap \mathbf{R}_{8_{+}}^{9}$. Choose coordinates $\left(\theta, x_{1}, x\right) \in S^{1} \times D^{8}$ and assume that $\phi$ is such that $\phi(\theta, 0, x) \in$ 
$\mathrm{R}_{9+}^{9} \cap \mathrm{R}_{8_{+}}^{9}, p_{8}\left(\phi\left(\theta, x_{1}, x\right)\right)=x_{1}$, where $p_{j}$ is the projection on the $j^{\text {th }}$ coordinate and that $p_{k} \phi\left(\theta,-x_{1}, x\right)=p_{k} \phi\left(\theta, x_{1}, x\right), k \neq 8$. There is then an embedding $\psi: S^{1} \times[0,1] \rightarrow \mathrm{R}_{9+}^{9} \quad$ such that $\psi\left(S^{1} \times 0\right) \subset \mathrm{R}_{9+}^{9} \cap \mathrm{R}_{9-}^{9}$ and $\psi\left(S^{1} \times 1\right)=$ $\phi\left(S^{1}, 1,0\right)$. Choose an embedding $\alpha: S^{1} \times D^{15} \rightarrow \mathrm{R}_{16+}^{16}$. Then there is an embedding $\beta: S^{1} \times I \rightarrow \mathrm{R}_{16+}^{16}$ that agrees with $\alpha(\partial B)$ in one end and maps the other to $\mathrm{R}_{16+}^{16} \cap \mathrm{R}_{16-}^{16}$. Let $f^{\prime}=\alpha g \phi$. Then we can extend $f^{\prime}$ to $\psi\left(S^{1} \times[0,1]\right)$ using $\beta$. Furthermore, $f^{\prime}$ induces a framing of $\alpha(\partial B)$ and this framing extends to a framing of $\beta\left(S^{1} \times I\right)$, which may be taken constant near the ends. Now, extend $f^{\prime}$ by $f^{\prime}\left(x,-x_{8}, x_{9}\right)=f^{\prime}\left(x, x_{8}, x_{9}\right)$ for $x_{9} \geq 0$. Using the framing of $\beta\left(S^{1} \times I\right)$ we may then extend $f^{\prime}$ to a tubular neighborhood of the "tube" where it is defined. Taking the double of this map we get a map $f^{\prime \prime}: N \rightarrow U$ where $N$ is a tubular neighborhood of $T^{2} \subset \mathrm{R}^{9} \subset S^{9}$ and $U$ is a tubular neighborhood of $K^{2} \subset \mathrm{R}^{16}$ such that $M_{f^{\prime \prime}}=K^{2}, \widetilde{M}_{f^{\prime \prime}}=T^{2}, f^{\prime \prime}: \partial N \hookrightarrow \partial U$. Using Theorems 3.1, 3.5 as in the above examples we get the desired $f$.

\section{REFERENCES}

1. A. Haefliger, Differentiable imbeddings, Bull. Amer. Math. Soc. 67 (1961), 109-112.

2. A. Haefliger, Knotted (4k-1)-spheres in 6k-space, Ann. of Math. 78 (1963), 501-526.

3. A. Haefliger, Plongements différentiable dans le domain stable, Comment. Math. Helv. 37 (1962), 155-176.

4. M. W. Hirsch, Immersions of manifolds, Trans. Amer. Math. Soc. 93 (1959), 242-276.

5. J. F. P. Hudson, Concordance, isotopy, and diffeotopy, Ann. of Math. 91 (1970), 425-448.

6. J. F. P. Hudson, Piecewise linear embeddings, Ann. of Math. 85 (1967), 1-33.

7. D. Johnson, Spin structures and quadratic forms on surfaces, London Math. Soc. 22 (1980), 365-373.

8. R. C. Kirby and L. R. Taylor, Pin structures on low-dimensional manifolds, in "Geometry of low-dimenssional manifolds: 2”, London Math. Soc. Lecture Notes Ser. 151 (1990), 177-242.

9. W. S. Massey, Proof of a Conjecture of Whitney, Pacific J. Math. 31 (1961), 143-156.

10. Y. Matsumoto, An elementary proof of Rohlin's signature theorem and its extension by Guillou and Marin, in "A la Recherche de la Topologie Perdue", edited by Guillou and Marin, Birkhäuser (1986), 119-139.

11. J. W. Milnor, Remarks Concerning Spin Manifolds, in "Differential and Combinatorial Topology”, edited by Cairns, Princeton University Press (1965), 55-62.

DEPARTMENT OF MATHEMATICS

UPPSALA UNIVERSITY

S-751 06 UPPSALA

SWEDEN

Email address : tobias@math.uu.se 